\title{
Boundedness of the Riesz potential in central Morrey-Orlicz spaces
}

\author{
Evgeniya Burtseva ${ }^{1}\left(\right.$ [) $\cdot$ Lech Maligranda ${ }^{1,2}$ (1) $\cdot$ Katsuo Matsuoka $^{3}(-)$
}

Received: 13 April 2021 / Accepted: 16 November 2021 / Published online: 23 February 2022

(c) The Author(s) 2022

\begin{abstract}
Boundedness of the maximal operator and the Calderón-Zygmund singular integral operators in central Morrey-Orlicz spaces were proved in papers (Maligranda et al. in Colloq Math 138:165-181, 2015; Maligranda et al. in Tohoku Math J 72:235-259, 2020) by the second and third authors. The weak-type estimates have also been proven. Here we show boundedness of the Riesz potential in central Morrey-Orlicz spaces and the corresponding weak-type version.
\end{abstract}

Keywords Riesz potential · Orlicz functions - Orlicz spaces · Morrey-Orlicz spaces · Central Morrey-Orlicz spaces · Weak central Morrey-Orlicz spaces

Mathematics Subject Classification Primary 46E30 - 42B20; Secondary 42B35

\section{Orlicz spaces and central Morrey-Orlicz spaces}

First of all, we recall the definition of Orlicz spaces on $\mathbb{R}^{n}$ and some of their properties to be used later on (see [24] and [26] for details).

Evgeniya Burtseva

evgeniya.burtseva@1tu.se

Lech Maligranda

lech.maligranda@put.poznan.pl

Katsuo Matsuoka

katsu.m@nihon-u.ac.jp

1 Department of Engineering Sciences and Mathematics, Luleå University of Technology, SE-971 87 Luleå, Sweden

2 Institute of Mathematics, Poznań University of Technology, ul. Piotrowo 3a, 60-965 Poznań, Poland

3 College of Economics, Nihon University, 1-3-2 Misaki-cho, Kanda, Chiyoda-ku, Tokyo 101-8360, Japan 
A function $\Phi:[0, \infty) \rightarrow[0, \infty)$ is called an Orlicz function, if it is ancreasing continuous and convex function with $\Phi(0)=0$. Each such a function $\Phi$ has an integral representation $\Phi(u)=\int_{0}^{u} \Phi_{+}^{\prime}(t) d t$, where the right-derivative $\Phi_{+}^{\prime}$ is a nondecreasing right-continuous function (see [24, Theorem 1.1]). We will write below estimates for everywhere differentiable Orlicz function $\Phi$, but then using the above integral representation, these estimates will be true for almost all $u>0$ with its right-derivative $\Phi_{+}^{\prime}$ instead of derivative $\Phi^{\prime}$. Of course, we have estimates

$$
\Phi(u) \leq u \Phi^{\prime}(u) \leq \Phi(2 u) \text { for all } u>0
$$

If we want to include in the Orlicz spaces, for example, spaces $L^{\infty}\left(\mathbb{R}^{n}\right), L^{p}\left(\mathbb{R}^{n}\right) \cap$ $L^{\infty}\left(\mathbb{R}^{n}\right)$ and $L^{p}\left(\mathbb{R}^{n}\right)+L^{\infty}\left(\mathbb{R}^{n}\right)$ for $1 \leq p<\infty$, then we need to consider a broader class of functions than Orlicz functions, the so-called Young functions. A function $\Phi:[0, \infty) \rightarrow[0, \infty]$ is called a Young function, if it is a nondecreasing convex function with $\lim _{u \rightarrow 0^{+}} \Phi(u)=\Phi(0)=0$, and not identically 0 or $\infty$ in $(0, \infty)$. It may have jump up to $\infty$ at some point $u>0$, but then it should be left continuous at $u$.

Let $(\Omega, \Sigma, \mu)$ be a $\sigma$-finite complete nonatomic measure space and $L^{0}(\Omega)$ be the space of all $\mu$-equivalent classes of real-valued and $\Sigma$-measurable functions defined on $\Omega$.

For any Young function $\Phi$, the Orlicz space $L^{\Phi}(\Omega)$, which contains all $f \in L^{0}(\Omega)$ such that $\int_{\Omega} \Phi(\varepsilon|f(x)|) d \mu(x)<\infty$ for some $\varepsilon=\varepsilon(f)>0$ with the LuxemburgNakano norm

$$
\|f\|_{L^{\Phi}}=\inf \left\{\varepsilon>0: \int_{\Omega} \Phi\left(\frac{|f(x)|}{\varepsilon}\right) d \mu(x) \leq 1\right\},
$$

is a Banach space (cf. [24, pp. 70-71], [26, pp. 15-16], [27, pp. 125-127] and [38, pp. $67-68])$. The fundamental function of the Orlicz space $L^{\Phi}(\Omega)$ is

$$
\varphi_{L^{\Phi}(\Omega)}(t)=\left\|\chi_{A}\right\|_{L^{\Phi}(\Omega)}=\left\|\chi_{[0, \mu(A)]}\right\|_{L^{\Phi}([0, \infty))}=1 / \Phi^{-1}(1 / t),
$$

where $\chi_{A}$ is the characteristic function of the set $A \subset \Omega, t=\mu(A)$ and $\Phi^{-1}$ is the right-continuous inverse of $\Phi$ defined by $\Phi^{-1}(v)=\inf \{u \geq 0: \Phi(u)>v\}$ with inf $\emptyset=\infty$.

To each Young function $\Phi$ one can associate another convex function $\Phi^{*}$, i.e., the complementary function to $\Phi$, which is defined by

$$
\Phi^{*}(v)=\sup _{u>0}[u v-\Phi(u)] \text { for } v \geq 0 .
$$

Then $\Phi^{*}$ is also a Young function and $\Phi^{* *}=\Phi$. Note that $u \leq \Phi^{-1}(u) \Phi^{*^{-1}}(u) \leq 2 u$ for all $u>0$.

We say that a Young function $\Phi$ satisfies the $\Delta_{2}$-condition and we write shortly $\Phi \in \Delta_{2}$, if $0<\Phi(u)<\infty$ for $u>0$ and there exists a constant $D_{2} \geq 1$ such that

$$
\Phi(2 u) \leq D_{2} \Phi(u) \text { for all } u>0
$$


In this paper we consider Orlicz spaces $L^{\Phi}\left(\mathbb{R}^{n}\right)$ on $\mathbb{R}^{n}$ with the Lebesgue measure. Then we define the Morrey-Orlicz spaces $M^{\Phi, \lambda}\left(\mathbb{R}^{n}\right)$ and central Morrey-Orlicz spaces $M^{\Phi, \lambda}(0)$. In the 2000s, several authors (for example, F. Deringoz, V. S. Guliyev, J. J. Hasanov, T. Mizuhara, E. Nakai, S. Samko, Y. Sawano, H. Tanaka and others) defined Orlicz versions of the Morrey space, i.e., Morrey-Orlicz spaces, and investigated the boundedness for the Hardy-Littlewood maximal operator and other operators on them (see, for example, [11, 19, 20, 34, 39] and the references therein). The Orlicz version of central Morrey spaces, i.e., central Morrey-Orlicz spaces were defined in papers by the second and third authors. They investigated boundedness on central Morrey-Orlicz spaces of the Hardy-Littlewood maximal operator in paper [28] and also boundedness of the Calderón-Zygmund singular integral operators on them in paper [29]. In this paper we present conditions under which the Riesz potential is bounded on central Morrey-Orlicz spaces.

For any Young function $\Phi$, number $\lambda \in \mathbb{R}$, a set $A \subset \mathbb{R}^{n}$ with $0<|A|<\infty$ and for $f \in L^{0}\left(\mathbb{R}^{n}\right)$ let

$$
\|f\|_{\Phi, \lambda, A}=\inf \left\{\varepsilon>0: \frac{1}{|A|^{\lambda}} \int_{A} \Phi\left(\frac{|f(x)|}{\varepsilon}\right) d x \leq 1\right\}
$$

and the corresponding (smaller) expression

$$
\|f\|_{\Phi, \lambda, A, \infty}=\inf \left\{\varepsilon>0: \sup _{u>0} \Phi\left(\frac{u}{\varepsilon}\right) \frac{1}{|A|^{\lambda}} d\left(f \chi_{A}, u\right) \leq 1\right\},
$$

where $d(f, u)=\left|\left\{x \in \mathbb{R}^{n}:|f(x)|>u\right\}\right|$. Note that $\|f\|_{\Phi, \lambda, A, \infty} \leq\|f\|_{\Phi, \lambda, A}$ provided that the expression on the right is finite. In fact, if $\|f\|_{\Phi, \lambda, A}<c$, then for arbitrary $u>0$ we have

$$
\begin{aligned}
1 & \geq \frac{1}{|A|^{\lambda}} \int_{A} \Phi\left(\frac{|f(x)|}{c}\right) d x \geq \frac{1}{|A|^{\lambda}} \int_{\{x \in A:|f(x)|>u\}} \Phi\left(\frac{|f(x)|}{c}\right) d x \\
& \geq \frac{1}{|A|^{\lambda}} \Phi\left(\frac{u}{c}\right) d\left(f \chi_{A}, u\right),
\end{aligned}
$$

and $\|f\|_{\Phi, \lambda, A, \infty} \leq c$. Hence, $\|f\|_{\Phi, \lambda, A, \infty} \leq\|f\|_{\Phi, \lambda, A}$.

Using these notions and considering open balls $B\left(x_{0}, r\right)$ with a center at $x_{0} \in \mathbb{R}^{n}$ and radius $r>0$, i.e. $B\left(x_{0}, r\right)=\left\{x \in \mathbb{R}^{n}:\left|x-x_{0}\right|<r\right\}$, and also open balls $B(0, r)=B_{r}$ with a center at 0 we can define Morrey-Orlicz spaces $M^{\Phi, \lambda}\left(\mathbb{R}^{n}\right)$ and weak Morrey-Orlicz spaces $W M^{\Phi, \lambda}\left(\mathbb{R}^{n}\right)$ :

$$
M^{\Phi, \lambda}\left(\mathbb{R}^{n}\right)=\left\{f \in L_{l o c}^{1}\left(\mathbb{R}^{n}\right):\|f\|_{M^{\Phi, \lambda}}=\sup _{x_{0} \in \mathbb{R}^{n}, r>0}\|f\|_{\Phi, \lambda, B\left(x_{0}, r\right)}<\infty\right\}
$$

and

$$
W M^{\Phi, \lambda}\left(\mathbb{R}^{n}\right)=\left\{f \in L_{l o c}^{1}\left(\mathbb{R}^{n}\right):\|f\|_{W M^{\Phi, \lambda}}=\sup _{x_{0} \in \mathbb{R}^{n}, r>0}\|f\|_{\Phi, \lambda, B\left(x_{0}, r\right), \infty}<\infty\right\} .
$$


Similarly, we can define central Morrey-Orlicz spaces $M^{\Phi, \lambda}(0)$ and weak central Morrey-Orlicz spaces $W M^{\Phi, \lambda}(0)$ :

$$
M^{\Phi, \lambda}(0)=\left\{f \in L_{l o c}^{1}\left(\mathbb{R}^{n}\right):\|f\|_{M^{\Phi, \lambda}(0)}=\sup _{r>0}\|f\|_{\Phi, \lambda, B_{r}}<\infty\right\}
$$

and

$$
W M^{\Phi, \lambda}(0)=\left\{f \in L_{l o c}^{1}\left(\mathbb{R}^{n}\right):\|f\|_{W M^{\Phi, \lambda}(0)}=\sup _{r>0}\|f\|_{\Phi, \lambda, B_{r}, \infty}<\infty\right\} .
$$

All these spaces are Banach ideal spaces on $\mathbb{R}^{n}$ (sometimes they are $\{0\}$, that is, they contain only all functions equivalent to 0 on $\mathbb{R}^{n}$ ). Moreover, we have continuous embeddings $M^{\Phi, \lambda}\left(\mathbb{R}^{n}\right) \stackrel{1}{\hookrightarrow} W M^{\Phi, \lambda}\left(\mathbb{R}^{n}\right), M^{\Phi, \lambda}(0) \stackrel{1}{\hookrightarrow} W M^{\Phi, \lambda}(0)$ and also $M^{\Phi, \lambda}\left(\mathbb{R}^{n}\right) \stackrel{1}{\hookrightarrow} M^{\Phi, \lambda}(0), W M^{\Phi, \lambda}\left(\mathbb{R}^{n}\right) \stackrel{1}{\hookrightarrow} W M^{\Phi, \lambda}(0)$.

Let us recall that the normed subspace $X=\left(X,\|\cdot\|_{X}\right)$ of $L^{0}(\Omega)$ is an ideal space on $\Omega$ : if $f, g \in X$ with $|f(x)| \leq|g(x)|$ for $\mu$-almost all $x \in \Omega$, and $g \in X$, then $f \in X$ and $\|f\|_{X} \leq\|g\|_{X}$. Here and further, for two Banach ideal spaces $X$ and $Y$, we use the symbol $X \hookrightarrow Y$ rather than $X \subset Y$ for continuous embedding. Moreover, the symbol $X \stackrel{C}{\hookrightarrow} Y$ indicates that $X \hookrightarrow Y$ with the norm of the embedding operator not bigger than $C$, i.e., $\|f\|_{Y} \leq C\|f\|_{X}$ for all $f \in X$.

Note that Morrey-Orlicz spaces and central Morrey-Orlicz spaces are generalizations of Orlicz spaces and Morrey spaces (on $\mathbb{R}^{n}$ ). In particular, we can obtain the following spaces (see [28] for more details):

(i) (Orlicz and weak Orlicz spaces) If $\lambda=0$, then

$$
\begin{aligned}
M^{\Phi, 0}\left(\mathbb{R}^{n}\right) & =M^{\Phi, 0}(0)=L^{\Phi}\left(\mathbb{R}^{n}\right) \text { and } \\
W M^{\Phi, 0}\left(\mathbb{R}^{n}\right) & =W M^{\Phi, 0}(0)=W L^{\Phi}\left(\mathbb{R}^{n}\right) .
\end{aligned}
$$

(ii) (Beurling-Orlicz and weak Beurling-Orlicz spaces) If $\lambda=1$, then

$$
M^{\Phi, 1}\left(\mathbb{R}^{n}\right)=B^{\Phi}\left(\mathbb{R}^{n}\right) \text { and } W M^{\Phi, 1}\left(\mathbb{R}^{n}\right)=W B^{\Phi}\left(\mathbb{R}^{n}\right)
$$

As for $B^{\Phi}\left(\mathbb{R}^{n}\right)$ and $W B^{\Phi}\left(\mathbb{R}^{n}\right)$, see [28].

(iii) (Classical Morrey, weak Morrey, central Morrey and weak central Morrey spaces) If $\Phi(u)=u^{p}, 1 \leq p<\infty$ and $\lambda \in \mathbb{R}$, then $M^{\Phi, \lambda}\left(\mathbb{R}^{n}\right)=$ $M^{p, \lambda}\left(\mathbb{R}^{n}\right), W M^{\Phi, \lambda}\left(\mathbb{R}^{n}\right)=W M^{p, \lambda}\left(\mathbb{R}^{n}\right)$ and $M^{\Phi, \lambda}(0)=M^{p, \lambda}(0)$, $W M^{\Phi, \lambda}(0)=W M^{p, \lambda}(0)$.

Here $M^{p, \lambda}\left(\mathbb{R}^{n}\right), W M^{p, \lambda}\left(\mathbb{R}^{n}\right), M^{p, \lambda}(0), W M^{p, \lambda}(0)$ are the classical Morrey, weak Morrey, central Morrey and weak central Morrey spaces, respectively.

We want to note that $M^{p, \lambda}\left(\mathbb{R}^{n}\right) \neq\{0\}$ if and only if $0 \leq \lambda \leq 1$ (see [6, Lemma $1]$ ) and $M^{p, \lambda}(0) \neq\{0\}$ if and only if $\lambda \geq 0$ (see [4, 6, 7]). Moreover, $M^{p, 0}\left(\mathbb{R}^{n}\right)=$ $M^{p, 0}(0)=L^{p}\left(\mathbb{R}^{n}\right)$ and $M^{p, 1}\left(\mathbb{R}^{n}\right)=L^{\infty}\left(\mathbb{R}^{n}\right)$ (see [25, Theorem 4.3.6]). However, $L^{\infty}\left(\mathbb{R}^{n}\right) \stackrel{1}{\hookrightarrow} M^{p, 1}(0)$ and the inclusion is strict. For example, in one-dimensional case 
$f(x)=\sum_{n=0}^{\infty} 2^{n / p} \chi_{\left[n, n+2^{-n}\right]}(|x|) \in M^{p, 1}(0) \backslash L^{\infty}\left(\mathbb{R}^{1}\right)$. Of course, for $0 \leq \lambda \leq 1$ the inclusion $M^{p, \lambda}\left(\mathbb{R}^{n}\right) \stackrel{1}{\hookrightarrow} M^{p, \lambda}(0)$ holds and is strict for $0<\lambda \leq 1$ (a suitable example we can find in [22, p. 156]). It is also true that if $1 \leq p<q<\infty, 0 \leq \mu<\lambda<1$ and $\frac{1-\lambda}{p}=\frac{1-\mu}{q}$, then

$$
M^{q, \mu}\left(\mathbb{R}^{n}\right) \stackrel{1}{\hookrightarrow} M^{p, \lambda}\left(\mathbb{R}^{n}\right) \text { and } M^{q, \mu}(0) \stackrel{1}{\hookrightarrow} M^{p, \lambda}(0) .
$$

Both inclusions are proper (see, for example, [21]); the second embedding in (8) is also true for $1<\lambda<\mu$. The embeddings (8) follow by the Hölder-Rogers inequality with $\frac{q}{p}>1$, since for any $x_{0} \in \mathbb{R}^{n}$ we have

$$
\begin{aligned}
\int_{B\left(x_{0}, r\right)}|f(x)|^{p} d x & \leq\left(\int_{B\left(x_{0}, r\right)}|f(x)|^{q} d x\right)^{p / q}\left|B\left(x_{0}, r\right)\right|^{1-p / q} \\
& =\left(\frac{1}{\left|B\left(x_{0}, r\right)\right|^{\mu}} \int_{B\left(x_{0}, r\right)}|f(x)|^{q} d x\right)^{p / q}\left|B\left(x_{0}, r\right)\right|^{1-p / q+\mu p / q} \\
& =\left(\frac{1}{\left|B\left(x_{0}, r\right)\right|^{\mu}} \int_{B\left(x_{0}, r\right)}|f(x)|^{q} d x\right)^{p / q}\left|B\left(x_{0}, r\right)\right|^{\lambda}
\end{aligned}
$$

and from the fact that $1-p / q+\mu p / q=(\mu-1) p / q+1=-(1-\lambda)+1=\lambda$.

If the supremum in definitions (4)-(7) is taken over all $r>1$, then we will have corresponding definitions of non-homogeneous Morrey-Orlicz spaces, nonhomogeneous weak Morrey-Orlicz spaces, non-homogeneous central Morrey-Orlicz spaces and non-homogeneous weak central Morrey-Orlicz spaces.

\section{The Riesz potential in Lebesgue, Orlicz and Morrey spaces}

The Riesz potential of order $\alpha \in(0, n)$ of a locally integrable function $f \in L^{0}\left(\mathbb{R}^{n}\right)$ is defined as

$$
I_{\alpha} f(x)=\int_{\mathbb{R}^{n}} \frac{f(y)}{|x-y|^{n-\alpha}} d y, \quad \text { for } x \in \mathbb{R}^{n} .
$$

The linear operator $I_{\alpha}$ plays a role in various branches of analysis, including potential theory, harmonic analysis, Sobolev spaces and partial differential equations. Therefore, investigations of the boundedness of the operator $I_{\alpha}$ between different spaces are important.

The classical Hardy-Littlewood-Sobolev theorem states that if $1<p<q<\infty$, then a Riesz potential $I_{\alpha}$ is of strong-type $(p, q)$, that is, bounded from $L^{p}\left(\mathbb{R}^{n}\right)$ to $L^{q}\left(\mathbb{R}^{n}\right)$ if and only if $1 / q=1 / p-\alpha / n$. For $p=1<q<\infty$ Zygmund proved that $I_{\alpha}$ is of weak-type $(1, q)$, that is, bounded from $L^{1}\left(\mathbb{R}^{n}\right)$ to $W L^{q}\left(\mathbb{R}^{n}\right)$, where $1 / q=1-\alpha / n$. The weak- $L^{q}$ space $W L^{q}\left(\mathbb{R}^{n}\right)=L^{q, \infty}\left(\mathbb{R}^{n}\right)$, called also the Marcinkiewicz space, consists of all $f \in L^{0}\left(\mathbb{R}^{n}\right)$ such that the quasi-norm $\|f\|_{q, \infty}=$ $\sup _{t>0} t\left|\left\{x \in \mathbb{R}^{n}:|f(x)|>t\right\}\right|^{1 / q}$ is finite. The proofs of these results we can find in 
the books [15, pp. 125-127], [16, pp. 2-5], [40, pp. 117-121], [41, pp. 150-154] and [42, pp. 86-87].

The boundedness of $I_{\alpha}$ from an Orlicz space $L^{\Phi}\left(\mathbb{R}^{n}\right)$ to another Orlicz space $L^{\Psi}\left(\mathbb{R}^{n}\right)$ was studied by Simonenko (1964), O’Neil (1965) and Torchinsky (1976) under some restrictions on the Orlicz functions $\Phi$ and $\Psi$. In 1999 Cianchi [10] gave a necessary and sufficient condition for the boundedness of $I_{\alpha}$ from $L^{\Phi}\left(\mathbb{R}^{n}\right)$ to $L^{\Psi}\left(\mathbb{R}^{n}\right)$ and from $L^{\Phi}\left(\mathbb{R}^{n}\right)$ to weak Orlicz space $W L^{\Psi}\left(\mathbb{R}^{n}\right)$. Another sufficient conditions for boundedness of the Riesz operator $I_{\alpha}$ (and even for a generalized fractional operator $I_{\rho}$ ) were given in 2001 by Nakai $[32,33]$. Then in 2017, Guliyev-Deringoz-Hasanov in [20, Theorem 3.3], gave more readable necessary and sufficient conditions for the boundedness of $I_{\alpha}$ from $L^{\Phi}\left(\mathbb{R}^{n}\right)$ to $W L^{\Psi}\left(\mathbb{R}^{n}\right)$ and from $L^{\Phi}\left(\mathbb{R}^{n}\right)$ to $L^{\Psi}\left(\mathbb{R}^{n}\right)$.

Results concerning boundedness of the Riesz potential between Morrey spaces were first obtained by Spanne with the Sobolev exponent $1 / q=1 / p-\alpha / n$, and this result was published in 1969 by Peetre [36]: if $0<\alpha<n, 1<p<n(1-\lambda) / \alpha, 0<$ $\lambda<1,1 / q=1 / p-\alpha / n$ and $\lambda / p=\mu / q$, then the Riesz potential $I_{\alpha}$ is bounded from $M^{p, \lambda}\left(\mathbb{R}^{n}\right)$ to $M^{q, \mu}\left(\mathbb{R}^{n}\right)$. Then in 1975 a stronger result was obtained by Adams [1], and reproved by Chiarenza-Frasca [9]. Adams proved boundedness of $I_{\alpha}$ from $M^{p, \lambda}\left(\mathbb{R}^{n}\right)$ to $M^{q_{1}, \lambda}\left(\mathbb{R}^{n}\right)$ with a better exponent $q_{1}$, namely $1 / q_{1}=1 / p-\alpha /[n(1-\lambda)]$. Adams result is stronger than the Peetre-Spanne theorem because $q<q_{1}$ and $(1-\mu) / q=$ $(1-\lambda) / q_{1}$, from which follows the embedding $M^{q_{1}, \lambda}\left(\mathbb{R}^{n}\right) \stackrel{1}{\hookrightarrow} M^{q, \mu}\left(\mathbb{R}^{n}\right)$ and this means that the target space $M^{q_{1}, \lambda}\left(\mathbb{R}^{n}\right)$ is smaller than target space $M^{q, \mu}\left(\mathbb{R}^{n}\right)$ in the Peetre-Spanne result. Central Morrey spaces $M^{p, \lambda}(0)$ were first introduced in [14, $\mathrm{p}$. 607] and in [2, p. 5] (see also [8, p. 257] and [13, p. 500] for $\lambda=1$ ). Further studies of the central Morrey spaces and their generalizations were investigated, for example, in $[6,17,18,37]$.

Result on the boundedness of the Riesz potential in these spaces was proved by $\mathrm{Fu}-$ Lin-Lu [12, Proposition 1.1]: if $1<p<n(1-\lambda) / \alpha, 0<\lambda<1,1 / q=1 / p-\alpha / n$ and $\lambda / p=\mu / q$, then the Riesz potential $I_{\alpha}$ is bounded from $M^{p, \lambda}(0)$ to $M^{q, \mu}(0)$ (see also [5, 17, 18]). Komori-Furuya and Sato [23, Proposition 1] showed that Adams type result on boundedness in central Morrey spaces does not hold. They showed that if $\frac{1-\mu}{q}=\frac{1-\lambda}{p}-\frac{\alpha}{n}$ and $\alpha / n<1 / p-1 / q<\alpha /[n(1-\lambda)]$, then $I_{\alpha}$ is not bounded from $M^{p, \lambda}(0)$ to $M^{q, \mu}(0)$ because $\mu / q=\lambda / p-(1 / p-\alpha / n-1 / q)<\lambda / p$.

We will generalize the last results to central Morrey-Orlicz spaces. In Theorem 2 , the necessary conditions for boundedness of $I_{\alpha}$ are given, and in Theorem 3 -sufficient conditions are presented.

In the proof of boundedness of the Riesz potential in the central Morrey-Orlicz spaces we will need some necessary estimates. We will present them in the next section.

\section{Some technical results}

To prove the main results of this paper, we need some technical calculations. In order not to hide the main ideas in proofs of the main results we collect such calculations in Lemma 1 below. 
Lemma 1 Let $\Phi$ be a Young function, $\Phi^{*}$ its complementary function, $0 \leq \lambda \leq 1$ and $r>0$. Then

(i) $\int_{B_{r}}|f(x) g(x)| d x \leq 2\left|B_{r}\right|^{\lambda}\|f\|_{\Phi, \lambda, B_{r}}\|g\|_{\Phi^{*}, \lambda, B_{r}}$.

(ii) $\left\|\chi_{B\left(x_{0}, r_{0}\right)}\right\|_{\Phi^{*}, \lambda, B_{r}} \leq \frac{\left|B_{r} \cap B\left(x_{0}, r_{0}\right)\right|}{\left|B_{r}\right|^{\lambda}} \Phi^{-1}\left(\frac{\left|B_{r}\right|^{\lambda}}{\left|B_{r} \cap B\left(x_{0}, r_{0}\right)\right|}\right)$, where $B_{r} \cap B\left(x_{0}, r_{0}\right) \neq$ $\emptyset$ for $x_{0} \in \mathbb{R}^{n}$ and $r_{0}>0$.

In particular, $\left\|\chi_{B_{r}}\right\|_{\Phi^{*}, \lambda, B_{r}} \leq \frac{\Phi^{-1}\left(\left|B_{r}\right|^{\lambda-1}\right)}{\left|B_{r}\right|^{\lambda-1}}$.

(iii) $\left\|\chi_{B_{t}}\right\|_{\Phi, \lambda, B_{r}}=1 / \Phi^{-1}\left(\frac{\left|B_{r}\right|^{\lambda}}{\left|B_{r} \cap B_{t}\right|}\right)$ and $\left\|\chi_{B_{t}}\right\|_{M^{\Phi, \lambda}(0)}=\frac{1}{\Phi^{-1}\left(\left|B_{t}\right|^{\lambda-1}\right)}$ for any $t>0$.

(iv) $\left\|\chi_{B_{t}}\right\|_{\Phi, \lambda, B_{r}, \infty}=1 / \Phi^{-1}\left(\frac{\left|B_{r}\right|^{\lambda}}{\left|B_{r} \cap B_{t}\right|}\right)$ and $\left\|\chi_{B_{t}}\right\|_{W M^{\Phi, \lambda}(0)}=\frac{1}{\Phi^{-1}\left(\left|B_{t}\right|^{\lambda-1}\right)}$ for any $t>0$.

Proof (i) This estimate was proved in [29, Lemma 2.6].

(ii) Since for $u>0$ we have $\Phi^{*}\left(\frac{u}{\Phi^{-1}(u)}\right) \leq u$ (cf. Lemma 2.6 in [29]) it follows for $u=\frac{\left|B_{r}\right|^{\lambda}}{\left|B_{r} \cap B\left(x_{0}, r_{0}\right)\right|}$ that

$$
\begin{aligned}
& \int_{B_{r}} \Phi^{*}\left(\frac{\chi_{B\left(x_{0}, r_{0}\right)}(x)\left|B_{r}\right|^{\lambda}}{\Phi^{-1}\left(\frac{\left|B_{r}\right|^{\lambda}}{\left|B_{r} \cap B\left(x_{0}, r_{0}\right)\right|}\right)\left|B_{r} \cap B\left(x_{0}, r_{0}\right)\right|}\right) d x \\
& \quad=\int_{B_{r} \cap B\left(x_{0}, r_{0}\right)} \Phi^{*}\left(\frac{\left|B_{r}\right|^{\lambda}}{\Phi^{-1}\left(\frac{\left|B_{r}\right|^{\lambda}}{\left|B_{r} \cap B\left(x_{0}, r_{0}\right)\right|}\right)\left|B_{r} \cap B\left(x_{0}, r_{0}\right)\right|}\right) d x \\
& \leq \frac{\left|B_{r}\right|^{\lambda}}{\left|B_{r} \cap B\left(x_{0}, r_{0}\right)\right|} \int_{B_{r} \cap B\left(x_{0}, r_{0}\right)} d x=\left|B_{r}\right|^{\lambda} .
\end{aligned}
$$

Hence, $\left\|\chi_{B\left(x_{0}, r_{0}\right)}\right\|_{\Phi^{*}, \lambda, B_{r}} \leq \Phi^{-1}\left(\frac{\left|B_{r}\right|^{\lambda}}{\left|B_{r} \cap B\left(x_{0}, r_{0}\right)\right|}\right) \frac{\left|B_{r} \cap B\left(x_{0}, r_{0}\right)\right|}{\left|B_{r}\right|^{\lambda}}$, and (ii) follows.

(iii) Let $t>0$. Since $\Phi\left(\Phi^{-1}(u)\right) \leq u$ for any $u>0$ it follows that

$$
\begin{aligned}
& \int_{B_{r}} \Phi\left(\chi_{B_{t}}(x) \Phi^{-1}\left(\frac{\left|B_{r}\right|^{\lambda}}{\left|B_{r} \cap B_{t}\right|}\right)\right) d x=\int_{B_{r} \cap B_{t}} \Phi\left(\Phi^{-1}\left(\frac{\left|B_{r}\right|^{\lambda}}{\left|B_{r} \cap B_{t}\right|}\right)\right) d x \\
& \quad \leq \int_{B_{r} \cap B_{t}} \frac{\left|B_{r}\right|^{\lambda}}{\left|B_{r} \cap B_{t}\right|} d x=\left|B_{r}\right|^{\lambda},
\end{aligned}
$$

and so $\left\|\chi_{B_{t}}\right\|_{\Phi, \lambda, B_{r}} \leq 1 / \Phi^{-1}\left(\frac{\left|B_{r}\right|^{\lambda}}{\left|B_{r} \cap B_{t}\right|}\right)$. On the other hand,

$$
1 \geq \frac{1}{\left|B_{r}\right|^{\lambda}} \int_{B_{r}} \Phi\left(\frac{\chi_{B_{t}}(x)}{\left\|\chi_{B_{t}}\right\|_{\Phi, \lambda, B_{r}}}\right) d x=\Phi\left(\frac{1}{\left\|\chi_{B_{t}}\right\|_{\Phi, \lambda, B_{r}}}\right) \frac{\left|B_{r} \cap B_{t}\right|}{\left|B_{r}\right|^{\lambda}}
$$


or

$$
\frac{\left|B_{r}\right|^{\lambda}}{\left|B_{r} \cap B_{t}\right|} \geq \Phi\left(\frac{1}{\left\|\chi_{B_{t}}\right\|_{\Phi, \lambda, B_{r}}}\right) .
$$

Since $u \leq \Phi^{-1}(\Phi(u))$ for any $u>0$ such that $\Phi(u)<\infty$ we obtain

$$
\Phi^{-1}\left(\frac{\left|B_{r}\right|^{\lambda}}{\left|B_{r} \cap B_{t}\right|}\right) \geq \Phi^{-1}\left(\Phi\left(\frac{1}{\left\|\chi_{B_{t}}\right\|_{\Phi, \lambda, B_{r}}}\right)\right) \geq \frac{1}{\left\|\chi_{B_{t}}\right\|_{\Phi, \lambda, B_{r}}},
$$

which together with the previous estimate gives equality $\left\|\chi_{B_{t}}\right\|_{\Phi, \lambda, B_{r}}=1 / \Phi^{-1}\left(\frac{\left|B_{r}\right|^{\lambda}}{\left|B_{r} \cap B_{t}\right|}\right)$. Thus,

$$
\begin{aligned}
\left\|\chi_{B_{t}}\right\|_{M^{\Phi, \lambda}(0)} & =\sup _{r>0}\left\|\chi_{B_{t}}\right\| \Phi, \lambda, B_{r}=\sup _{r>0} \frac{1}{\Phi^{-1}\left(\frac{\left|B_{r}\right|^{\lambda}}{\left|B_{r} \cap B_{t}\right|}\right)} \\
& =\max \left[\sup _{r \leq t} \frac{1}{\Phi^{-1}\left(\frac{\left|B_{r}\right|^{\lambda}}{\left|B_{r} \cap B_{t}\right|}\right)}, \sup _{r \geq t} \frac{1}{\Phi^{-1}\left(\frac{\left|B_{r}\right|^{\lambda}}{\left|B_{r} \cap B_{t}\right|}\right)}\right] \\
& =\max \left[\sup _{r \leq t} \frac{1}{\Phi^{-1}\left(\left|B_{r}\right|^{\lambda-1}\right)}, \sup _{r \geq t} \frac{1}{\Phi^{-1}\left(\frac{\left|B_{r}\right|^{\lambda}}{\left|B_{t}\right|}\right)}\right]=\frac{1}{\Phi^{-1}\left(\left|B_{t}\right|^{\lambda-1}\right)},
\end{aligned}
$$

and point (iii) of the lemma has been proved.

(iv) For $t>0$ we have

$$
\begin{aligned}
& \sup _{u>0} \Phi\left(\frac{u}{\varepsilon}\right) \frac{1}{\left|B_{r}\right|^{\lambda}}\left|\left\{x \in B_{r}: \chi_{B_{t}}(x)>u\right\}\right| \\
& \quad=\sup _{0<u<1} \Phi\left(\frac{u}{\varepsilon}\right) \frac{\left|B_{r} \cap B_{t}\right|}{\left|B_{r}\right|^{\lambda}}=\Phi\left(\frac{1}{\varepsilon}\right) \frac{\left|B_{r} \cap B_{t}\right|}{\left|B_{r}\right|^{\lambda}} .
\end{aligned}
$$

Thus,

$$
\begin{aligned}
\left\|\chi_{B_{t}}\right\|_{\Phi, \lambda, B_{r}, \infty} & =\inf \left\{\varepsilon>0: \Phi\left(\frac{1}{\varepsilon}\right) \frac{\left|B_{r} \cap B_{t}\right|}{\left|B_{r}\right|^{\lambda}} \leq 1\right\} \\
& \leq \inf \left\{\varepsilon>0: \frac{1}{\varepsilon} \leq \Phi^{-1}\left(\frac{\left|B_{r}\right|^{\lambda}}{\left|B_{r} \cap B_{t}\right|}\right)\right\} \leq 1 / \Phi^{-1}\left(\frac{\left|B_{r}\right|^{\lambda}}{\left|B_{r} \cap B_{t}\right|}\right),
\end{aligned}
$$

because $1 / \varepsilon \leq \Phi^{-1}(\Phi(1 / \varepsilon))$. On the other hand, since $1 \geq \Phi\left(\frac{1}{\left\|\chi_{B_{t}}\right\|_{\Phi, \lambda, B_{r}, \infty}}\right) \frac{\left|B_{r} \cap B_{t}\right|}{\left|B_{r}\right|^{\lambda}}$ it follows that

$$
\frac{1}{\left\|\chi_{B_{t}}\right\|_{\Phi, \lambda, B_{r}, \infty}} \leq \Phi^{-1}\left(\Phi\left(\frac{1}{\left\|\chi_{B_{t}}\right\|_{\Phi, \lambda, B_{r}, \infty}}\right)\right) \leq \Phi^{-1}\left(\frac{\left|B_{r}\right|^{\lambda}}{\left|B_{r} \cap B_{t}\right|}\right),
$$

which together gives the first equality in (iv). The second equality in (iv) has the same proof as the second equality in (iii). 


\section{On the norm of the dilation operator in central Morrey-Orlicz spaces}

For any $a>0$ and $x \in \mathbb{R}^{n}$ we define the dilation operator $D_{a}$ by

$$
D_{a} f(x)=f(a x), \quad f \in L^{0}\left(\mathbb{R}^{n}\right) .
$$

The dilation operator is bounded in central Morrey-Orlicz spaces $M^{\Phi, \lambda}(0)$ and we will calculate its norm. For this purpose quantity $s_{\Phi^{-1}}$ is needed for the Orlicz function $\Phi:$

$$
s_{\Phi^{-1}}(t)=\sup _{s>0} \frac{\Phi^{-1}(s t)}{\Phi^{-1}(s)}, \quad t>0 .
$$

Theorem 1 If $\Phi$ is an Orlicz function, $0 \leq \lambda \leq 1$ and $a>0$, then the operator norm of $D_{a}$ is

$$
\left\|D_{a}\right\|_{M^{\Phi, \lambda}(0) \rightarrow M^{\Phi, \lambda}(0)}=s_{\Phi^{-1}}\left(a^{n(\lambda-1)}\right) .
$$

Proof By definition of $s_{\Phi^{-1}}$, for any $s>0, a>0$, we have

$$
\Phi^{-1}\left(a^{n(\lambda-1)} s\right) \leq s_{\Phi^{-1}}\left(a^{n(\lambda-1)}\right) \Phi^{-1}(s)
$$

and so

$$
\Phi\left(\frac{\Phi^{-1}\left(a^{n(\lambda-1)} s\right)}{s_{\Phi^{-1}}\left(a^{n(\lambda-1)}\right)}\right) \leq \Phi\left(\Phi^{-1}(s)\right)=s .
$$

For $a^{n(\lambda-1)} s=\Phi(u)$ we have $u=\Phi^{-1}\left(a^{n(\lambda-1)} s\right)$ and

$$
\Phi\left(\frac{u}{s_{\Phi^{-1}}\left(a^{n(\lambda-1)}\right)}\right) \leq a^{n(1-\lambda)} \Phi(u), \quad \text { for any } u>0
$$

Therefore, from (12) it follows that for any $f \in M^{\Phi, \lambda}(0)$ and $r>0$,

$$
\begin{gathered}
\int_{B_{r}} \Phi\left(\frac{\left|D_{a} f(x)\right|}{s_{\Phi^{-1}}\left(a^{n(\lambda-1)}\right)\|f\|_{M^{\Phi, \lambda}(0)}}\right) d x \\
=\int_{B_{r}} \Phi\left(\frac{|f(a x)|}{s_{\Phi^{-1}}\left(a^{n(\lambda-1)}\right)\|f\|_{M^{\Phi, \lambda}(0)}}\right) d x \\
=a^{-n} \int_{B_{a r}} \Phi\left(\frac{|f(y)|}{s_{\Phi^{-1}}\left(a^{n(\lambda-1)}\right)\|f\|_{M^{\Phi, \lambda}(0)}}\right) d y
\end{gathered}
$$




$$
\begin{aligned}
& \leq a^{-n} a^{n(1-\lambda)} \int_{B_{a r}} \Phi\left(\frac{|f(y)|}{\|f\|_{M^{\Phi, \lambda}(0)}}\right) d y \leq a^{-\lambda n}\left|B_{a r}\right|^{\lambda} \\
& =a^{-\lambda n} v_{n}^{\lambda}(a r)^{\lambda n}=\left|B_{r}\right|^{\lambda},
\end{aligned}
$$

which means that $\left\|D_{a} f\right\|_{M^{\Phi, \lambda}(0)} \leq s_{\Phi^{-1}}\left(a^{n(\lambda-1)}\right)\|f\|_{M^{\Phi, \lambda}(0)}$. Here, $v_{n}=\left|B_{1}\right|$.

To show that (11) holds we consider the characteristic function $\chi_{B_{t}}(x)$ of the ball $B_{t}, t>0$. Note that $D_{a} \chi_{B_{t}}(x)=\chi_{B_{t / a}}(x)$. Moreover, by Lemma 1(iii) we get

$$
\begin{aligned}
\sup _{t>0} \frac{\left\|D_{a} \chi_{B_{t}}\right\|_{M^{\Phi, \lambda}(0)}}{\left\|\chi_{B_{t}}\right\|_{M^{\Phi, \lambda}(0)}} & =\sup _{t>0} \frac{\Phi^{-1}\left(\left|B_{t}\right|^{\lambda-1}\right)}{\Phi^{-1}\left(\left|B_{t / a}\right|^{\lambda-1}\right)}=\sup _{t>0} \frac{\Phi^{-1}\left(v_{n}^{\lambda-1} t^{n(\lambda-1)}\right)}{\Phi^{-1}\left(v_{n}^{\lambda-1}\left(\frac{t}{a}\right)^{n(\lambda-1)}\right)} \\
& =\sup _{s>0} \frac{\Phi^{-1}(s)}{\Phi^{-1}\left(a^{n(1-\lambda)} s\right)}=\sup _{s>0} \frac{\Phi^{-1}\left(s a^{n(\lambda-1)}\right)}{\Phi^{-1}(s)}=s_{\Phi^{-1}}\left(a^{n(\lambda-1)}\right) .
\end{aligned}
$$

This brings us to (11).

\section{The Riesz potential in central Morrey-Orlicz spaces-necessary conditions}

We begin to study the boundedness of the Riesz potential, first finding the necessary conditions for its boundedness.

Theorem 2 Let $0<\alpha<n, \Phi, \Psi$ be Orlicz functions and $0 \leq \lambda, \mu<1$.

(i) If the Riesz potential $I_{\alpha}$ is bounded from $M^{\Phi, \lambda}(0)$ to $M^{\Psi, \mu}(0)$, then there are positive constants $C_{1}, C_{2}$ such that

(a) $u^{\frac{\alpha}{n}} \Phi^{-1}\left(u^{\lambda-1}\right) \leq C_{1} \Psi^{-1}\left(u^{\mu-1}\right)$ for any $u>0$.

(b) $s_{\Psi^{-1}}\left(u^{\mu-1}\right) \leq C_{2} u^{\frac{\alpha}{n}} s_{\Phi^{-1}}\left(u^{\lambda-1}\right)$ for any $u>0$.

(ii) If there exists a small constant $c>0$ such that $c \leq \frac{v_{n}^{\lambda / \mu}}{v_{n-1}}$ with $v_{0}=1$ and

$$
\liminf _{t \rightarrow \infty} \frac{\Phi^{-1}\left(c t^{\lambda}\right)}{\Psi^{-1}\left(t^{\mu}\right)}=\infty
$$

then $I_{\alpha}$ is not bounded from $M^{\Phi, \lambda}(0)$ to $M^{\Psi, \mu}(0)$.

Proof (i) (a) Let $t>0$ and $x \in B_{t}$. In this case we have

$$
I_{\alpha} \chi_{B_{t}}(x)=\int_{B_{t}}|x-y|^{\alpha-n} d y \geq(2 t)^{\alpha-n}\left|B_{t}\right|=v_{n} 2^{\alpha-n} t^{\alpha}
$$

or

$$
t^{\alpha} \chi_{B_{t}}(x) \leq \frac{2^{n-\alpha}}{v_{n}} I_{\alpha} \chi_{B_{t}}(x) \chi_{B_{t}}(x)
$$


Then

$$
\left\|t^{\alpha} \chi_{B_{t}}\right\|_{M^{\Psi, \mu}(0)} \leq \frac{2^{n-\alpha}}{v_{n}}\left\|I_{\alpha} \chi_{B_{t}}\right\|_{M^{\Psi, \mu}(0)} \leq \frac{2^{n-\alpha}}{v_{n}} C\left\|\chi_{B_{t}}\right\|_{M^{\Phi, \lambda}(0)},
$$

and by the Lemma 1 (iii) we obtain

$$
\frac{t^{\alpha}}{\Psi^{-1}\left(\left|B_{t}\right|^{\mu-1}\right)} \leq \frac{2^{n-\alpha}}{v_{n}} C \frac{1}{\Phi^{-1}\left(\left|B_{t}\right|^{\lambda-1}\right)}
$$

which means

$$
\frac{t^{\alpha}}{\Psi^{-1}\left(v_{n}^{\mu-1} t^{(\mu-1) n}\right)} \leq \frac{2^{n-\alpha}}{v_{n}} \frac{C}{\Phi^{-1}\left(v_{n}^{\lambda-1} t^{(\lambda-1) n}\right)}
$$

Thus,

$$
t^{\alpha / n} \Phi^{-1}\left(v_{n}^{\lambda-1} t^{\lambda-1}\right) \leq \frac{2^{n-\alpha}}{v_{n}} C \Psi^{-1}\left(v_{n}^{\mu-1} t^{\mu-1}\right),
$$

which by a simple change of variables can be rewritten as

$$
u^{\alpha / n} \Phi^{-1}\left(u^{\lambda-1}\right) \leq C_{1} \Psi^{-1}\left(u^{\mu-1}\right) \text { for any } u>0,
$$

where $C_{1}=2^{n-\alpha} v_{n}^{\alpha / n-1} C$.

(i) (b) First, note that we have identity

$$
I_{\alpha}\left(D_{t} f\right)(x)=t^{-\alpha} D_{t}\left(I_{\alpha} f\right)(x) \text { for any } t>0 .
$$

In fact,

$$
I_{\alpha}\left(D_{t} f\right)(x)=\int_{\mathbb{R}^{n}} \frac{f(t y)}{|x-y|^{n-\alpha}} d y=t^{-\alpha} \int_{\mathbb{R}^{n}} \frac{f(y)}{|y-t x|^{n-\alpha}} d y=t^{-\alpha} D_{t}\left(I_{\alpha} f\right)(x) .
$$

Now, let $f \in M^{\Phi, \lambda}(0)$. Using the above identity and applying Theorem 1 we obtain

$$
\left\|I_{\alpha}\left(D_{t} f\right)\right\|_{M^{\Psi, \mu}(0)}=t^{-\alpha}\left\|D_{t}\left(I_{\alpha} f\right)\right\|_{M^{\Psi, \mu}(0)}=t^{-\alpha} s_{\Psi^{-1}}\left(t^{n(\mu-1)}\right)\left\|I_{\alpha} f\right\|_{M^{\Psi, \mu}(0)} .
$$

Assumption of boundedness of $I_{\alpha}$ and reuse of Theorem 1 gives

$$
\begin{aligned}
\left\|I_{\alpha} f\right\|_{M^{\Psi, \mu}(0)} & =\frac{t^{\alpha}}{s_{\Psi^{-1}}\left(t^{n(\mu-1)}\right)}\left\|I_{\alpha}\left(D_{t} f\right)\right\|_{M^{\Psi, \mu}(0)} \\
& \leq \frac{t^{\alpha}}{s_{\Psi^{-1}}\left(t^{n(\mu-1)}\right)} C\left\|D_{t} f\right\|_{M^{\Phi, \lambda}(0)} \\
& =C \frac{t^{\alpha}}{s_{\Psi^{-1}}\left(t^{n(\mu-1)}\right)} s_{\Phi^{-1}}\left(t^{n(\lambda-1)}\right)\|f\|_{M^{\Phi, \lambda}(0)},
\end{aligned}
$$


or

$$
\left\|I_{\alpha} f\right\|_{M^{\Psi, \mu}(0)} \leq C \frac{u^{\alpha / n} s_{\Phi^{-1}}\left(u^{\lambda-1}\right)}{s_{\Psi^{-1}}\left(u^{\mu-1}\right)}\|f\|_{M^{\Phi, \lambda}(0)} \text { for any } u>0 \text {. }
$$

Thus,

$$
\left\|I_{\alpha} f\right\|_{M^{\Psi, \mu}(0)} \leq C \inf _{u>0} \frac{u^{\alpha / n} s_{\Phi^{-1}}\left(u^{\lambda-1}\right)}{s_{\Psi^{-1}}\left(u^{\mu-1}\right)}\|f\|_{M^{\Phi, \lambda}(0)} .
$$

We must have that $\inf _{u>0} \frac{u^{\alpha / n} s_{\Phi^{-1}}\left(u^{\lambda-1}\right)}{s_{\Psi^{-1}}\left(u^{\mu-1}\right)}=c>0$ since otherwise $I_{\alpha} f=0$ and we get a contradiction. Therefore,

$$
s_{\Psi^{-1}}\left(u^{\mu-1}\right) \leq \frac{C}{c} u^{\alpha / n} s_{\Phi^{-1}}\left(u^{\lambda-1}\right) \text { for any } u>0 .
$$

(ii) We follow the same argument as in [23, Proposition 1]. Let $R \geq 1, x_{R}=$ $(R, 0, \ldots, 0) \in \mathbb{R}^{n}$ and $f_{R}(x)=\chi_{B\left(x_{R}, 1\right)}(x)$. Then

$$
\begin{aligned}
\left\|f_{R}\right\|_{M^{\Phi, \lambda}(0)} & =\operatorname{supinf}_{r>0}\left\{\varepsilon>0: \frac{1}{\left|B_{r}\right|^{\lambda}} \int_{B_{r}} \Phi\left(\frac{\chi_{B\left(x_{R}, 1\right)(x)}}{\varepsilon}\right) d x \leq 1\right\} \\
& =\operatorname{supinf}_{r>0}\left\{\varepsilon>0: \frac{1}{\left|B_{r}\right|^{\lambda}} \int_{B_{r} \cap B\left(x_{R}, 1\right)} \Phi\left(\frac{1}{\varepsilon}\right) d x \leq 1\right\} \\
& =\operatorname{supinf}_{r>0}\left\{\varepsilon>0: \frac{\left|B_{r} \cap B\left(x_{R}, 1\right)\right|}{\left|B_{r}\right|^{\lambda}} \Phi\left(\frac{1}{\varepsilon}\right) \leq 1\right\} \\
& =\sup _{r>R-1} \inf \left\{\varepsilon>0: \frac{\left|B_{r} \cap B\left(x_{R}, 1\right)\right|}{\left|B_{r}\right|^{\lambda}} \Phi\left(\frac{1}{\varepsilon}\right) \leq 1\right\},
\end{aligned}
$$

because if $0<r \leq R-1$ then $\left|B_{r} \cap B\left(x_{R}, 1\right)\right|=0$. Thus,

$$
\left\|f_{R}\right\|_{M^{\Phi, \lambda}(0)}=\sup _{r>R-1} \frac{1}{\Phi^{-1}\left(\frac{\left|B_{r}\right|^{\lambda}}{\left|B_{r} \cap B\left(x_{R}, 1\right)\right|}\right)} .
$$

We will consider two cases: $R-1<r<R$ and $r \geq R$. In the first case, using calculations from [6, p. 161], we can prove that for $n \geq 2$

$$
\left|B_{r} \cap B\left(x_{R}, 1\right)\right| \leq 2^{\frac{n}{2}} v_{n-1}\left(\frac{r}{R}\right)^{n},
$$

and so

$$
\frac{\left|B_{r}\right|^{\lambda}}{\left|B_{r} \cap B\left(x_{R}, 1\right)\right|} \geq \frac{v_{n}^{\lambda} r^{\lambda n} R^{n}}{2^{\frac{n}{2}} v_{n-1} r^{n}} \geq \frac{v_{n}^{\lambda}}{2^{\frac{n}{2}} v_{n-1}} R^{\lambda n}>\frac{v_{n}^{\lambda}}{2^{n} v_{n-1}} R^{\lambda n}
$$


For $n=1$ and $R-1<r<R$ with $v_{0}=1$ we have

$$
\begin{aligned}
\frac{\left|B_{r}\right|^{\lambda}}{\left|B_{r} \cap B\left(x_{R}, 1\right)\right|} & =\frac{(2 r)^{\lambda}}{r-R+1}=\frac{2^{\lambda} r^{\lambda-1}}{1-\frac{R-1}{r}}>\frac{2^{\lambda} R^{\lambda-1}}{1-\frac{R-1}{r}} \\
& =\frac{2^{\lambda} R^{\lambda}}{R-\frac{R(R-1)}{r}}>2^{\lambda} R^{\lambda}=v_{1}^{\lambda} R^{\lambda}>\frac{v_{1}^{\lambda}}{2 v_{0}} R^{\lambda}
\end{aligned}
$$

In the second case, $\left|B_{r} \cap B\left(x_{R}, 1\right)\right| \leq\left|B\left(x_{R}, 1\right)\right|=v_{n}$ and

$$
\frac{\left|B_{r}\right|^{\lambda}}{\left|B_{r} \cap B\left(x_{R}, 1\right)\right|} \geq \frac{v_{n}^{\lambda} r^{\lambda n}}{v_{n}} \geq v_{n}^{\lambda-1} R^{\lambda n}
$$

Thus,

$$
\left\|f_{R}\right\|_{M^{\Phi, \lambda}(0)} \leq \max \left[\frac{1}{\Phi^{-1}\left(\frac{v_{n}^{\lambda}}{2^{n} v_{n-1}} R^{\lambda n}\right)}, \frac{1}{\Phi^{-1}\left(v_{n}^{\lambda-1} R^{\lambda n}\right)}\right]
$$

Since $\frac{v_{n-1}}{v_{n}} \geq \sqrt{\frac{n}{2 \pi}}$ with $v_{0}=1$ (see [3, Theorem 2]), it follows that $\frac{2^{n} v_{n-1}}{v_{n}} \geq 1$ and then $\frac{v_{n}^{\lambda}}{2^{n} v_{n-1}} \leq v_{n}^{\lambda-1}$, which gives

$$
\left\|f_{R}\right\|_{M^{\Phi, \lambda}(0)} \leq \frac{1}{\Phi^{-1}\left(\frac{v_{n}^{\lambda}}{2^{n} v_{n-1}} R^{\lambda n}\right)}
$$

Next, we will estimate $I_{\alpha} f_{R}$. If $x, y \in B\left(x_{R}, 1\right)$ then $|x-y| \leq 2$ and we obtain

$$
\begin{aligned}
I_{\alpha} f_{R}(x) & =\int_{\mathbb{R}^{n}} \frac{\chi_{B\left(x_{R}, 1\right)}(y)}{|x-y|^{n-\alpha}} d y=\int_{B\left(x_{R}, 1\right)}|x-y|^{\alpha-n} d y \\
& \geq 2^{\alpha-n}\left|B\left(x_{R}, 1\right)\right| \chi_{B\left(x_{R}, 1\right)}(x)=2^{\alpha-n} v_{n} \chi_{B\left(x_{R}, 1\right)}(x) .
\end{aligned}
$$

Thus,

$$
\begin{aligned}
\left\|I_{\alpha} f_{R}\right\|_{M^{\Psi, \mu}(0)} & =\sup _{r>0}\left\|I_{\alpha} f_{R}\right\|_{\Psi, \mu, B_{r}} \geq\left\|I_{\alpha} f_{R}\right\|_{\Psi, \mu, B_{R+1}} \\
& =\inf \left\{\varepsilon>0: \int_{B_{R+1}} \Psi\left(\frac{\left|I_{\alpha} f_{R}(x)\right|}{\varepsilon}\right) d x \leq\left|B_{R+1}\right|^{\mu}\right\} .
\end{aligned}
$$

Since $x \in B\left(x_{R}, 1\right)$ and $B_{R+1} \cap B\left(x_{R}, 1\right)=B\left(x_{R}, 1\right)$ it follows that

$$
\left\|I_{\alpha} f_{R}\right\|_{M^{\Psi, \mu}(0)} \geq \inf \left\{\varepsilon>0: \int_{B\left(x_{R}, 1\right) \cap B_{R+1}} \Psi\left(2^{\alpha-n} v_{n} / \varepsilon\right) d x \leq\left|B_{R+1}\right|^{\mu}\right\}
$$




$$
\begin{aligned}
& =\frac{2^{\alpha-n} v_{n}}{\Psi^{-1}\left(\frac{\left|B_{R+1}\right|^{\mu}}{\left|B\left(x_{R}, 1\right)\right|}\right)}=\frac{2^{\alpha-n} v_{n}}{\Psi^{-1}\left(v_{n}^{\mu-1}(R+1)^{\mu n}\right)} \\
& \geq \frac{2^{\alpha-n} v_{n}}{\Psi^{-1}\left(v_{n}^{\mu-1} 2^{\mu n} R^{\mu n}\right)} .
\end{aligned}
$$

Making the substitution $t^{\mu}=v_{n}^{\mu-1} 2^{\mu n} R^{\mu n}$ we obtain

$$
\begin{aligned}
\frac{\left\|I_{\alpha} f_{R}\right\|_{M^{\Psi, \mu}(0)}}{\left\|f_{R}\right\|_{M^{\Phi, \lambda}(0)}} \geq 2^{\alpha-n} v_{n} \frac{\Phi^{-1}\left(\frac{v_{n}^{\lambda}}{2^{n} v_{n-1}} R^{\lambda n}\right)}{\Psi^{-1}\left(v_{n}^{\mu-1} 2^{\mu n} R^{\mu n}\right)}=2^{\alpha-n} v_{n} \frac{\Phi^{-1}\left(\frac{v_{n}^{\frac{\lambda}{\mu}}}{2^{n+\lambda n} v_{n-1}} t^{\lambda}\right)}{\Psi^{-1}\left(t^{\mu}\right)} \\
\\
\geq \frac{2^{\alpha-n} v_{n}}{2^{n+\lambda n}} \frac{\Phi^{-1}\left(\frac{v_{n}^{\frac{\lambda}{\mu}}}{v_{n-1}^{\lambda}} t^{\lambda}\right)}{\Psi^{-1}\left(t^{\mu}\right)} \geq 2^{\alpha-2 n-\lambda n} v_{n} \frac{\Phi^{-1}\left(c t^{\lambda}\right)}{\Psi^{-1}\left(t^{\mu}\right)}
\end{aligned}
$$

and

$$
\liminf _{R \rightarrow \infty} \frac{\left\|I_{\alpha} f_{R}\right\|_{M^{\Psi, \mu}(0)}}{\left\|f_{R}\right\|_{M^{\Phi, \lambda}(0)}} \geq 2^{\alpha-2 n-\lambda n} v_{n} \liminf _{t \rightarrow \infty} \frac{\Phi^{-1}\left(c t^{\lambda}\right)}{\Psi^{-1}\left(t^{\mu}\right)}=\infty
$$

Thus, the operator $I_{\alpha}$ is not bounded from $M^{\Phi, \lambda}(0)$ to $M^{\Psi, \mu}(0)$.

\section{The Riesz potential in central Morrey-Orlicz spaces - sufficient conditions}

We want to prove boundedness of the Riesz potential $I_{\alpha}$ between two different central Morrey-Orlicz spaces. The following lemmas are important for proving the main result.

Lemma 2 Let $0<\alpha<n, \Phi$ be an Orlicz function and $0 \leq \lambda<1$. If $f \in M^{\Phi, \lambda}(0)$, then there exists a constant $C_{3}>0$ such that

$$
\int_{\mathbb{R}^{n} \backslash B_{r}} \frac{|f(y)|}{|y|^{n-\alpha}} d y \leq C_{3}\|f\|_{M^{\Phi, \lambda}(0)} \int_{\left|B_{r}\right|}^{\infty} t^{\alpha / n} \Phi^{-1}\left(t^{\lambda-1}\right) \frac{d t}{t}
$$

for all $r>0$.

Proof We prove this lemma using the same arguments as in the proof of Theorem 7.1 in [35] and Lemma 2.5 in [29]. From the Lemma 1 (i) and (ii) it follows that

$$
\int_{\mathbb{R}^{n} \backslash B_{r}} \frac{|f(y)|}{|y|^{n-\alpha}} d y=\sum_{j=1}^{\infty} \int_{B_{2^{j_{r}}} \backslash B_{2^{j-1} 1_{r}}} \frac{|f(y)|}{|y|^{n-\alpha}} d y \leq \sum_{j=1}^{\infty} \frac{1}{\left(2^{j-1} r\right)^{n-\alpha}} \int_{B_{2^{j} j_{r}}}|f(y)| d y
$$




$$
\begin{aligned}
& =2^{n-\alpha} v_{n}^{1-\alpha / n} \sum_{j=1}^{\infty} \frac{1}{\left|B_{2^{j_{r}}}\right|^{1-\alpha / n}} \int_{B_{2 j_{r}}}|f(y)| d y \\
& \leq 2^{n-\alpha+1} v_{n}^{1-\alpha / n} \sum_{j=1}^{\infty}\left|B_{2^{j} r}\right|^{\lambda-1+\alpha / n}\|f\|_{\Phi, \lambda, B_{2} j_{r}}\|1\|_{\Phi^{*}, \lambda, B_{2} j_{r}} \\
& \leq C_{3}^{\prime} \sum_{j=1}^{\infty}\left|B_{2 j_{r}}\right|^{\lambda-1+\alpha / n}\|f\|_{\Phi, \lambda, B_{2} j_{r}} \frac{\Phi^{-1}\left(\left|B_{2^{j_{r}}}\right|^{\lambda-1}\right)}{\left|B_{2^{j_{r}}}\right|^{\lambda-1}} \\
& =\frac{C_{3}^{\prime}}{n \ln 2}\|f\|_{M^{\Phi, \lambda}(0)} \sum_{j=1}^{\infty}\left|B_{2^{j_{r}}}\right|^{\alpha / n} \Phi^{-1}\left(\left|B_{2^{j}}\right|^{\lambda-1}\right) \int_{\left|B_{2^{j-1} r_{r}}\right|}^{\left|B_{2^{j_{r}}}\right|} \frac{d t}{t} \\
& \leq \frac{C_{3}^{\prime}}{n \ln 2} 2^{\alpha}\|f\|_{M^{\Phi, \lambda}(0)} \sum_{j=1}^{\infty} \int_{\left|B_{2 j-1}\right|}^{\left|B_{2 j_{r}}\right|} t^{\alpha / n} \Phi^{-1}\left(t^{\lambda-1}\right) \frac{d t}{t} \\
& \leq C_{3}\|f\|_{M^{\Phi, \lambda}(0)} \int_{\left|B_{r}\right|}^{\infty} t^{\alpha / n} \Phi^{-1}\left(t^{\lambda-1}\right) \frac{d t}{t},
\end{aligned}
$$

where $C_{3}^{\prime}=2^{n-\alpha+1} v_{n}^{1-\alpha / n}$ and $C_{3}=\frac{2^{\alpha}}{n \ln 2} C_{3}^{\prime}$. Thus, we arrive to the assertion of Lemma 2.

Next, we show the following well-definedness of $I_{\alpha} f$ when $f \in M^{\Phi, \lambda}(0)$.

Lemma 3 Let $0<\alpha<n, \Phi$ be an Orlicz function and $0 \leq \lambda<1$. If the integral $\int_{\left|B_{r}\right|}^{\infty} t^{\alpha / n} \Phi^{-1}\left(t^{\lambda-1}\right) \frac{d t}{t}$ is convergent for any $r>0$ and $f \in M^{\Phi, \lambda}(0)$ then the Riesz potential $I_{\alpha} f$ is well-defined.

Proof We will prove this lemma using the same arguments that were presented in the proof in [30, Theorem 2.1]. Let $f \in M^{\Phi, \lambda}(0), r>0$ and $x \in B_{r}$, and let

$$
I_{\alpha} f(x)=I_{\alpha}\left(f \chi_{B_{2 r}}\right)(x)+I_{\alpha}\left(f\left(1-\chi_{B_{2 r}}\right)\right)(x) .
$$

Since $f \chi_{B_{2 r}} \in L^{1}\left(\mathbb{R}^{n}\right)$, the first term is well-defined. Indeed, in view of [31, Theorem 1.1 , Chapter 2] the requirement $I_{\alpha}\left|f \chi_{B_{2 r}}\right| \not \equiv \infty$ for any $f \in M^{\Phi, \lambda}(0)$ and $r>0$ is equivalent to

$$
\int_{B_{2 r}}(1+|y|)^{\alpha-n}|f(y)| d y<\infty
$$

The last inequality is true since $\left\|(1+|y|)^{\alpha-n}\right\|_{\Phi^{*}, \lambda, B_{2 r}} \leq \frac{(1+2 r)^{\alpha-n}}{\left(\Phi^{*}\right)^{-1}\left(\left|B_{2 r}\right|^{\lambda-1}\right)}$ and by Lemma 1 (i) we obtain

$$
\begin{aligned}
\int_{B_{2 r}}(1+|y|)^{\alpha-n}|f(y)| d y & \leq 2\left|B_{2 r}\right|^{\lambda}\|f\|_{\Phi, \lambda, B_{2 r}}\left\|(1+|y|)^{\alpha-n}\right\|_{\Phi^{*}, \lambda, B_{2 r}} \\
& \leq 2\left|B_{2 r}\right|^{\lambda} \frac{(1+2 r)^{\alpha-n}}{\left(\Phi^{*}\right)^{-1}\left(\left|B_{2 r}\right|^{\lambda-1}\right)}\|f\|_{M^{\Phi, \lambda}(0)}<\infty .
\end{aligned}
$$


For the second term for any $x \in B_{r}$ we have

$$
\left|I_{\alpha}\left(f\left(1-\chi_{B_{2 r}}\right)\right)(x)\right| \leq \int_{\mathbb{R}^{n} \backslash B_{2 r}} \frac{|f(y)|}{|x-y|^{n-\alpha}} d y \leq 2^{n-\alpha} \int_{\mathbb{R}^{n} \backslash B_{2 r}} \frac{|f(y)|}{|y|^{n-\alpha}} d y .
$$

Since the integral $\int_{\left|B_{r}\right|}^{\infty} t^{\alpha / n} \Phi^{-1}\left(t^{\lambda-1}\right) \frac{d t}{t}$ is convergent for any $r>0$ and $f \in M^{\Phi, \lambda}(0)$ it follows from Lemma 2 that $I_{\alpha}\left(f\left(1-\chi_{B_{2 r}}\right)\right)(x)$ is well-defined for all $x \in B_{r}$.

Further, since for $0<s<r$,

$$
f \chi_{B_{2 s}}+f\left(1-\chi_{B_{2 s}}\right)=f \chi_{B_{2 r}}+f\left(1-\chi_{B_{2 r}}\right)
$$

it follows that for $x \in B_{s} \subset B_{r}$,

$$
I_{\alpha}\left(f \chi_{B_{2 s}}\right)(x)+I_{\alpha}\left(f\left(1-\chi_{B_{2 s}}\right)\right)(x)=I_{\alpha}\left(f \chi_{B_{2 r}}\right)(x)+I_{\alpha}\left(f\left(1-\chi_{B_{2 r}}\right)\right)(x) .
$$

This shows that $I_{\alpha} f$ is independent of $B_{r}$ containing $x$. Thus, $I_{\alpha} f$ is well-defined on $\mathbb{R}^{n}$.

Now we will present sufficient conditions on spaces so that the operator $I_{\alpha}$ is bounded between distinct central Morrey-Orlicz spaces. In the proofs of these estimates we will use estimates from [28] for the Hardy-Littlewood maximal operator. The Hardy-Littlewood maximal operator $M$ is defined for $f \in L_{l o c}^{1}\left(\mathbb{R}^{n}\right)$ and $x \in \mathbb{R}^{n}$ by

$$
M f(x)=\sup _{r>0} \frac{1}{|B(x, r)|} \int_{B(x, r)}|f(y)| d y .
$$

Then, for an Orlicz function $\Phi$ and $0 \leq \lambda \leq 1$, this operator $M$ is bounded on $M^{\Phi, \lambda}(0)$, provided $\Phi^{*} \in \Delta_{2}$, that is, there exists a constant $C_{0}>1$ such that

$$
\|M f\|_{M^{\Phi, \lambda}(0)} \leq C_{0}\|f\|_{M^{\Phi, \lambda}(0)} \text { for all } f \in M^{\Phi, \lambda}(0)
$$

(see [28, Theorem 6(i)]). Moreover, $M$ is bounded from $M^{\Phi, \lambda}(0)$ to $W M^{\Phi, \lambda}(0)$, that is, there exists a constant $c_{0}>1$ such that $\|M f\|_{W M^{\Phi, \lambda}(0)} \leq c_{0}\|f\|_{M^{\Phi, \lambda}(0)}$ for all $f \in M^{\Phi, \lambda}(0)$ (see [28, Theorem 6(ii)]).

Theorem 3 Let $0<\alpha<n, \Phi, \Psi$ be Orliczfunctions and either $0<\lambda, \mu<1, \lambda \neq \mu$ or $\lambda=\mu=0$. Assume that there exist constants $C_{4}, C_{5} \geq 1$ such that

$$
\int_{u}^{\infty} t^{\frac{\alpha}{n}} \Phi^{-1}\left(t^{\lambda-1}\right) \frac{d t}{t} \leq C_{4} \Psi^{-1}\left(u^{\mu-1}\right) \text { for all } u>0
$$

and

$$
\int_{u}^{\infty} t^{\frac{\alpha}{n}} \Phi^{-1}\left(\frac{r^{\lambda}}{t}\right) \frac{d t}{t} \leq C_{5} \Psi^{-1}\left(\frac{r^{\mu}}{u}\right) \text { for all } u>0 \text { and for all } r>0
$$


(i) If $\Phi^{*} \in \Delta_{2}$, then $I_{\alpha}$ is bounded from $M^{\Phi, \lambda}(0)$ to $M^{\Psi, \mu}(0)$, that is, there exists a constant $C_{6} \geq 1$ such that $\left\|I_{\alpha} f\right\|_{M^{\Psi, \mu}(0)} \leq C_{6}\|f\|_{M^{\Phi, \lambda}(0)}$ for all $f \in M^{\Phi, \lambda}(0)$.

(ii) The operator $I_{\alpha}$ is bounded from $M^{\Phi, \lambda}(0)$ to $W M^{\Psi, \mu}(0)$, that is, there exists a constant $c_{6} \geq 1$ such that $\left\|I_{\alpha} f\right\|_{W M^{\Psi, \mu}(0)} \leq c_{6}\|f\|_{M^{\Phi, \lambda}(0)}$ for all $f \in$ $M^{\Phi, \lambda}(0)$.

Remark 1 The same conclusions hold for non-homogeneous versions of $M^{\Phi, \lambda}(0)$ and $M^{\Psi, \mu}(0)$.

Remark 2 From the estimate (15) we get the inequality (a) in Theorem 2(i). Namely, using the concavity of the function $\Phi^{-1}$ we get

$$
\begin{aligned}
\int_{u}^{\infty} t^{\frac{\alpha}{n}} \Phi^{-1}\left(t^{\lambda-1}\right) \frac{d t}{t} & \geq \int_{u}^{2 u} t^{\frac{\alpha}{n}} \Phi^{-1}\left(t^{\lambda-1}\right) \frac{d t}{t} \geq u^{\frac{\alpha}{n}} \Phi^{-1}\left((2 u)^{\lambda-1}\right) \ln 2 \\
& \geq \frac{\ln 2}{2^{1-\lambda}} u^{\frac{\alpha}{n}} \Phi^{-1}\left(u^{\lambda-1}\right) .
\end{aligned}
$$

Remark 3 Note that if either $\lambda=\mu>0$ or $\lambda=0$ and $\mu>0$, then estimate (16) doesn't hold.

Remark 4 If $\lambda=\mu=0$, then inequalities (15) and (16) are the same. Moreover, condition (15) in this case is a sufficient condition for boundedness of $I_{\alpha}$ from Orlicz space $L^{\Phi}\left(\mathbb{R}^{n}\right)$ to weak Orlicz space $W L^{\Psi}\left(\mathbb{R}^{n}\right)$, and if additionally $\Phi^{*} \in \Delta_{2}$ then $I_{\alpha}$ is bounded from Orlicz space $L^{\Phi}\left(\mathbb{R}^{n}\right)$ to Orlicz space $L^{\Psi}\left(\mathbb{R}^{n}\right)$ (proof we can find, for example, in [20, Theorem 3.3]).

In the proof of Theorem 3 the following lemma plays a crucial role.

Lemma 4 Let $0<\alpha<n, \Phi, \Psi$ be Orlicz functions, $\Phi^{*} \in \Delta_{2}$ and either $0<\lambda, \mu<$ $1, \lambda \neq \mu$ or $\lambda=\mu=0$. If the estimate (16) holds, then there exists a constant $C_{7} \geq 1$ such that

$$
\int_{B_{r}} \Psi\left(\frac{\int_{B_{2 r}} \frac{|f(y)|}{|x-y|^{n-\alpha}} d y}{C_{7}\|f\|_{M^{\Phi, \lambda}(0)}}\right) d x \leq\left|B_{r}\right|^{\mu}, \quad \text { for all } f \in M^{\Phi, \lambda}(0) \text { and } r>0 .
$$

Proof Let $f \in M^{\Phi, \lambda}(0)$. We write $I_{\alpha}\left(f \chi_{B_{2 r}}\right)$ as follows

$$
\begin{aligned}
I_{\alpha}\left(f \chi_{B_{2 r}}\right)(x)= & \int_{B_{2 r}} \frac{|f(y)|}{|x-y|^{n-\alpha}} d y=\int_{|x-y| \leq \delta} \frac{\left|f(y) \chi_{B_{2 r}}(y)\right|}{|x-y|^{n-\alpha}} d y \\
& +\int_{|x-y|>\delta} \frac{\left|f(y) \chi_{B_{2 r}}(y)\right|}{|x-y|^{n-\alpha}} d y=: J_{1} f(x)+J_{2} f(x),
\end{aligned}
$$


where $\delta>0$ will be defined later on. It is known that

$$
J_{1} f(x) \leq C_{8}\left|B_{\delta}\right|^{\frac{\alpha}{n}} M\left(f \chi_{B_{2 r}}\right)(x)
$$

where $C_{8}=\frac{2^{\alpha}}{2^{\alpha}-1} C_{3}^{\prime}$. Note that for any parameters $u>0$ and $r>0$ we have

$$
\begin{aligned}
\int_{u}^{\infty} t^{\frac{\alpha}{n}} \Phi^{-1}\left(\frac{r^{\lambda}}{t}\right) \frac{d t}{t} & \geq \int_{u}^{2 u} t^{\frac{\alpha}{n}} \Phi^{-1}\left(\frac{r^{\lambda}}{t}\right) \frac{d t}{t} \\
& \geq \ln 2 u^{\frac{\alpha}{n}} \Phi^{-1}\left(\frac{r^{\lambda}}{2 u}\right) \geq \frac{\ln 2}{2} u^{\frac{\alpha}{n}} \Phi^{-1}\left(\frac{r^{\lambda}}{u}\right)
\end{aligned}
$$

Thus, applying (16) we obtain

$$
J_{1} f(x) \leq \frac{2}{\ln 2} C_{5} C_{8} \frac{\Psi^{-1}\left(\frac{\left|B_{2 r}\right|^{\mu}}{\left|B_{\delta}\right|}\right)}{\Phi^{-1}\left(\frac{\left|B_{2 r}\right|^{\lambda}}{\left|B_{\delta}\right|}\right)} M\left(f \chi_{B_{2 r}}\right)(x) .
$$

Following Hedberg's method we get for $J_{2} f(x)$

$$
\begin{aligned}
J_{2} f(x) & =\sum_{k=1}^{\infty} \int_{2^{k-1} \delta<|x-y| \leq 2^{k} \delta} \frac{\left|f(y) \chi_{B_{2 r}}(y)\right|}{|x-y|^{n-\alpha}} d y \\
& \leq \sum_{k=1}^{\infty}\left(2^{k-1} \delta\right)^{\alpha-n} \int_{|x-y| \leq 2^{k} \delta}\left|f(y) \chi_{B_{2 r}}(y)\right| d y \\
& =\sum_{k=1}^{\infty}\left(2^{k-1} \delta\right)^{\alpha-n} \int_{B_{2 r}}\left|f(y) \chi_{B\left(x, 2^{k} \delta\right)}(y)\right| d y .
\end{aligned}
$$

From Lemma 1 (i) and (ii) it follows that

$$
\begin{aligned}
J_{2} f(x) \leq & 2\left|B_{2 r}\right|^{\lambda}\|f\|_{\Phi, \lambda, B_{2 r}} \sum_{k=1}^{\infty}\left(2^{k-1} \delta\right)^{\alpha-n}\left\|\chi_{B\left(x, 2^{k} \delta\right)}\right\|_{\Phi^{*}, \lambda, B_{2 r}} \\
\leq & 2^{n-\alpha+1}\|f\|_{\Phi, \lambda, B_{2 r}} \sum_{k=1}^{\infty}\left(2^{k} \delta\right)^{\alpha-n}\left|B_{2 r} \cap B\left(x, 2^{k} \delta\right)\right| \\
& \cdot \Phi^{-1}\left(\frac{\left|B_{2 r}\right|^{\lambda}}{\left|B_{2 r} \cap B\left(x, 2^{k} \delta\right)\right|}\right) .
\end{aligned}
$$


Taking into account that $u \Phi^{-1}(1 / u)$ is increasing and $\left|B_{2 r} \cap B\left(x, 2^{k} \delta\right)\right| \leq\left|B\left(x, 2^{k} \delta\right)\right|$ we obtain

$$
\begin{aligned}
J_{2} f(x) & \leq 2^{n-\alpha+1}\|f\|_{\Phi, \lambda, B_{2 r}} \sum_{k=1}^{\infty}\left(2^{k} \delta\right)^{\alpha-n} \Phi^{-1}\left(\frac{\left|B_{2 r}\right|^{\lambda}}{\left|B\left(x, 2^{k} \delta\right)\right|}\right)\left|B\left(x, 2^{k} \delta\right)\right| \\
& =2^{n-\alpha+1} v_{n}\|f\|_{\Phi, \lambda, B_{2 r}} \sum_{k=1}^{\infty}\left(2^{k} \delta\right)^{\alpha} \Phi^{-1}\left(\frac{\left|B_{2 r}\right|^{\lambda}}{\left|B_{2^{k} \delta}\right|}\right) \\
& =\frac{C_{3}^{\prime}}{n \ln 2}\|f\|_{\Phi, \lambda, B_{2 r}} \sum_{k=1}^{\infty}\left|B_{2^{k} \delta}\right|^{\frac{\alpha}{n}} \Phi^{-1}\left(\frac{\left|B_{2 r}\right|^{\lambda}}{\left|B_{2^{k} \delta}\right|}\right)_{\left|B_{2^{k-1} \delta_{\delta}}\right|}^{\left|B_{2^{k} \delta}\right|} \frac{d t}{t} \\
& \leq \frac{C_{3}^{\prime}}{n \ln 2}\|f\|_{\Phi, \lambda, B_{2 r}} \sum_{k=1}^{\infty} \mid B_{\left.2^{k}\right|^{\mid \frac{\alpha}{n}}} \int_{\left|B_{2^{k}}\right|}^{\left|B_{2^{k-1}}\right|} \Phi^{-1}\left(\frac{\left|B_{2 r}\right|^{\lambda}}{t}\right) \frac{d t}{t} \\
& \leq C_{3}\|f\|_{\Phi, \lambda, B_{2 r}} \int_{\left|B_{\delta}\right|}^{\infty} t^{\frac{\alpha}{n}} \Phi^{-1}\left(\frac{\left|B_{2 r}\right|^{\lambda}}{t}\right) \frac{d t}{t} \\
& \leq C_{5} C_{3}\|f\|_{M^{\Phi, \lambda}(0)} \Psi^{-1}\left(\frac{\left|B_{2 r}\right|^{\mu}}{\left|B_{\delta}\right|}\right) .
\end{aligned}
$$

Now we choose $\delta>0$ such that

$$
\frac{M f(x)}{C_{0}\|f\|_{M^{\Phi, \lambda}(0)}}=\Phi^{-1}\left(\frac{\left|B_{2 r}\right|^{\lambda}}{\left|B_{\delta}\right|}\right)
$$

where the constant $C_{0}$ is from (14). Then

$$
J_{1} f(x) \leq \frac{2}{\ln 2} C_{5} C_{8} C_{0}\|f\|_{M^{\Phi, \lambda}(0)} \Psi^{-1}\left(\frac{\left|B_{2 r}\right|^{\mu}}{\left|B_{\delta}\right|}\right)
$$

and

$$
\begin{aligned}
& \int_{B_{2 r}} \frac{|f(y)|}{|x-y|^{n-\alpha}} d y=J_{1} f(x)+J_{2} f(x) \\
& \quad \leq\left(\frac{2}{\ln 2} C_{5} C_{8} C_{0}+C_{5} C_{3}\right)\|f\|_{M^{\Phi, \lambda}(0)} \Psi^{-1}\left(\frac{\left|B_{2 r}\right|^{\mu}}{\left|B_{\delta}\right|}\right)
\end{aligned}
$$

Thus, with $C_{9}=2 C_{5} \max \left(\frac{2}{\ln 2} C_{0} C_{8}, C_{3}\right)$ we obtain

$$
\int_{B_{2 r}} \frac{|f(y)|}{|x-y|^{n-\alpha}} d y \leq C_{9}\|f\|_{M^{\Phi, \lambda}(0)} \Psi^{-1}\left(\left|B_{2 r}\right|^{\mu-\lambda} \Phi\left(\frac{M f(x)}{C_{0}\|f\|_{M^{\Phi, \lambda}(0)}}\right)\right) .
$$


Then

$$
\begin{aligned}
\Psi\left(\frac{\int_{B_{2 r}} \frac{|f(y)|}{|x-y|^{n-\alpha}} d y}{C_{9}\|f\|_{M^{\Phi, \lambda}(0)}}\right) & \leq\left|B_{2 r}\right|^{\mu-\lambda} \Phi\left(\frac{M f(x)}{\|M f\|_{M^{\Phi, \lambda}(0)}}\right) \\
& =2^{n(\mu-\lambda)}\left|B_{r}\right|^{\mu-\lambda} \Phi\left(\frac{M f(x)}{\|M f\|_{M^{\Phi, \lambda}(0)}}\right) .
\end{aligned}
$$

Finally, with $C_{7}=2^{n(\mu-\lambda)} C_{9}$ we get

$$
\frac{1}{\left|B_{r}\right|^{\mu}} \int_{B_{r}} \Psi\left(\frac{\int_{B_{2 r}} \frac{|f(y)|}{|x-y|^{n-\alpha}} d y}{C_{7}\|f\|_{M^{\Phi, \lambda}(0)}}\right) d x \leq \frac{1}{\left|B_{r}\right|^{\lambda}} \int_{B_{r}} \Phi\left(\frac{M f(x)}{\|M f\|_{M^{\Phi, \lambda}(0)}}\right) d x \leq 1
$$

and we arrive to the statement of this lemma.

Proof of Theorem 3 (i) Let $0<\alpha<n$ and $0<\lambda<1,0<\mu<1$. Let also $f \in M^{\Phi, \lambda}(0)$ and $r>0$. Since $I_{\alpha} f$ is well-defined by Lemma 3 , we prove only that

$$
\left\|I_{\alpha} f\right\|_{M^{\Psi, \mu}(0)} \leq C_{6}\|f\|_{M^{\Phi, \lambda}(0)} .
$$

Now, by (13), for $C_{6}=2 \max \left(C_{7}, 2^{n-\alpha} C_{3} C_{4}\right)$, it follows that

$$
\begin{aligned}
\int_{B_{r}} & \Psi\left(\frac{\left|I_{\alpha} f(x)\right|}{C_{6}\|f\|_{M^{\Phi, \lambda}(0)}}\right) d x \\
& \leq \frac{1}{2} \int_{B_{r}} \Psi\left(\frac{\left|I_{\alpha}\left(f \chi_{B_{2 r}}\right)(x)\right|}{C_{7}\|f\|_{M^{\Phi, \lambda}(0)}}\right) d x+\frac{1}{2} \int_{B_{r}} \Psi\left(\frac{\left|I_{\alpha}\left(f\left(1-\chi_{B_{2 r}}\right)\right)(x)\right|}{2^{n-\alpha} C_{3} C_{4}\|f\|_{M^{\Phi, \lambda}(0)}}\right) d x \\
= & : \frac{1}{2}\left(I_{1}+I_{2}\right) .
\end{aligned}
$$

From Lemma 4 we get that $I_{1} \leq\left|B_{r}\right|^{\mu}$ for all $r>0$.

Next, we estimate $I_{2}$. Since for $x \in B_{r}$ and $|y| \geq 2 r$ we have $|x|<r \leq \frac{|y|}{2}$ and $|x-y| \geq|y|-|x|>\frac{|y|}{2}$, it follows that

$$
\left|I_{\alpha}\left(f\left(1-\chi_{B_{2 r}}\right)\right)(x)\right| \leq \int_{\mathbb{R}^{n} \backslash B_{2 r}} \frac{|f(y)|}{|x-y|^{n-\alpha}} d y \leq 2^{n-\alpha} \int_{\mathbb{R}^{n} \backslash B_{2 r}} \frac{|f(y)|}{|y|^{n-\alpha}} d y .
$$

By Lemma 2 and the estimate (15) we obtain

$$
\Psi\left(\frac{\int_{\mathbb{R}^{n} \backslash B_{2 r}} \frac{|f(y)|}{|y|^{n-\alpha}} d y}{C_{3} C_{4}\|f\|_{M^{\Phi, \lambda}(0)}}\right) d x \leq \Psi\left(\frac{1}{C_{4}} \int_{\left|B_{2 r}\right|} t^{\alpha / n} \Phi^{-1}\left(t^{\lambda-1}\right) \frac{d t}{t}\right)
$$




$$
\leq \Psi\left(\Psi^{-1}\left(\left|B_{2 r}\right|^{\mu-1}\right)\right) \leq\left|B_{2 r}\right|^{\mu-1} .
$$

Thus, for $x \in B_{r}$

$$
I_{2} \leq \int_{B_{r}} \Psi\left(\frac{\int_{\mathbb{R}^{n} \backslash B_{2 r}} \frac{|f(y)|}{|y|^{n-\alpha}} d y}{C_{3} C_{4}\|f\|_{M^{\Phi, \lambda}(0)}}\right) d x \leq\left|B_{2 r}\right|^{\mu-1} \cdot\left|B_{r}\right|<\left|B_{r}\right|^{\mu}
$$

Hence,

$$
\frac{1}{\left|B_{r}\right|^{\mu}} \int_{B_{r}} \Psi\left(\frac{\left|I_{\alpha} f(x)\right|}{C_{6}\|f\|_{M^{\Phi, \lambda}(0)}}\right) d x<1
$$

and so

$$
\left\|I_{\alpha} f\right\|_{M^{\Psi, \mu}(0)} \leq C_{6}\|f\|_{M^{\Phi, \lambda}(0)} .
$$

(ii) Similarly to the previous case, by (13), we obtain for $u>0$

$$
\begin{aligned}
& \Psi\left(\frac{\left|I_{\alpha} f(x)\right|}{c_{6}\|f\|_{M^{\Phi, \lambda}(0)}}\right) \\
& \quad \leq \frac{1}{2} \Psi\left(\frac{\left|I_{\alpha}\left(f \chi_{B_{2 r}}\right)(x)\right|}{c_{7}\|f\|_{M^{\Phi, \lambda}(0)}}\right)+\frac{1}{2} \Psi\left(\frac{\left|I_{\alpha}\left(f\left(1-\chi_{B_{2 r}}\right)\right)(x)\right|}{2^{n-\alpha+1} C_{3} C_{4}\|f\|_{M^{\Phi, \lambda}(0)}}\right) \\
& \quad=: \frac{1}{2}\left(I_{3}+I_{4}\right),
\end{aligned}
$$

with $c_{6}=2 \max \left(c_{7}, 2^{n-\alpha+1} C_{3} C_{4}\right), c_{7}=2^{n(\mu-\lambda)+1} c_{9}$ and $c_{9}=2 C_{5} \max \left(\frac{2}{\ln 2} c_{0}\right.$ $\left.C_{8}, C_{3}\right)$.

Since $\Psi(u) d(g, u)=v d\left(g, \Psi^{-1}(v)\right)=v d(\Psi(g), v)$ for any $u>0$ with $v=$ $\Psi(u)$ and

$$
d\left(\Psi\left(\frac{\left|I_{\alpha} f(x)\right|}{c_{6}\|f\|_{M^{\Phi, \lambda}(0)}}\right), u\right) \leq d\left(I_{3}, u\right)+d\left(I_{4}, u\right)
$$

it follows that

$$
\sup _{u>0} \frac{\Psi(u)}{\left|B_{r}\right|^{\mu}} d\left(\frac{\left|I_{\alpha} f(x)\right|}{c_{6}\|f\|_{M^{\Phi, \lambda}(0)}}, u\right) \leq \sup _{u>0} \frac{u}{\left|B_{r}\right|^{\mu}} d\left(I_{3}, u\right)+\sup _{u>0} \frac{u}{\left|B_{r}\right|^{\mu}} d\left(I_{4}, u\right) .
$$

From the proof of Lemma 4 for all $r>0$

$$
I_{3}=\Psi\left(\frac{\left|I_{\alpha}\left(f \chi_{B_{2 r}}\right)(x)\right|}{c_{7}\|f\|_{M^{\Phi, \lambda}(0)}}\right) \leq \frac{1}{2}\left|B_{r}\right|^{\mu-\lambda} \Phi\left(\frac{M f(x)}{\|M f\|_{W M^{\Phi, \lambda}(0)}}\right)
$$


and

$$
\begin{aligned}
\sup _{u>0} \frac{u}{\left|B_{r}\right|^{\mu}} d\left(I_{1}, u\right) & \leq \sup _{u>0} \frac{u}{\left|B_{r}\right|^{\mu}} d\left(\frac{1}{2}\left|B_{r}\right|^{\mu-\lambda} \Phi\left(\frac{M f(x)}{\|M f\|_{W M^{\Phi, \lambda}(0)}}\right), u\right) \\
& =\frac{1}{2} \sup _{u>0} \frac{u}{\left|B_{r}\right|^{\lambda}} d\left(\Phi\left(\frac{M f(x)}{\|M f\|_{W M^{\Phi, \lambda}(0)}}\right), u\right) \\
& =\frac{1}{2} \sup _{u>0} \frac{\Phi(u)}{\left|B_{r}\right|^{\lambda}} d\left(\frac{M f(x)}{\|M f\|_{W M^{\Phi, \lambda}(0)}}, u\right) \leq \frac{1}{2} .
\end{aligned}
$$

For $I_{4}$, using Lemma 2 we obtain

$$
I_{4}=\Psi\left(\frac{\left|I_{\alpha}\left(f\left(1-\chi_{B_{2 r}}\right)\right)(x)\right|}{2^{n-\alpha+1} C_{3} C_{4}\|f\|_{M^{\Phi, \lambda}(0)}}\right) \leq \frac{1}{2}\left|B_{r}\right|^{\mu-1}
$$

and

$$
\sup _{u>0} \frac{u}{\left|B_{r}\right|^{\mu}} d\left(I_{4}, u\right) \leq \sup _{u>0} \frac{u}{\left|B_{r}\right|^{\mu}} d\left(\frac{1}{2}\left|B_{r}\right|^{\mu-1}, u\right)=\frac{1}{2} \sup _{u>0} u d\left(\frac{1}{\left|B_{r}\right|}, u\right) \leq \frac{1}{2} .
$$

Thus,

$$
\sup _{u>0} \frac{\Psi(u)}{\left|B_{r}\right|^{\mu}} d\left(\frac{\left|I_{\alpha} f(x)\right|}{c_{6}\|f\|_{M^{\Phi, \lambda}(0)}}, u\right) \leq 1
$$

and $\left\|I_{\alpha} f\right\|_{W M^{\Psi, \mu}(0)} \leq c_{6}\|f\|_{M^{\Phi, \lambda}(0)}$.

Example 1 Let $0<\alpha<n, 1<p<\frac{n(1-\lambda)}{\alpha}, 0 \leq \lambda<1$, and

$$
\Phi(u)=u^{p}, \quad \Psi(u)=u^{q} \text { with } 1<p<q<\infty .
$$

Then $\Phi^{*}(u)=(p-1) p^{-p^{\prime}} u^{p^{\prime}}$, where $1 / p+1 / p^{\prime}=1$ and $\Phi^{*}(2 u)=2^{p^{\prime}} \Phi^{*}(u)$, that is, $\Phi^{*} \in \Delta_{2}$. The estimate (15) holds since

$$
\int_{u}^{\infty} t^{\alpha / n} \Phi^{-1}\left(t^{\lambda-1}\right) \frac{d t}{t}=\int_{u}^{\infty} t^{\frac{\alpha}{n}+\frac{\lambda-1}{p}} \frac{d t}{t}=\frac{1}{\frac{1-\lambda}{p}-\frac{\alpha}{n}} u^{\frac{\alpha}{n}+\frac{\lambda-1}{p}}
$$

for all $u>0$, where the last integral is convergent because $p<\frac{n(1-\lambda)}{\alpha}$. If $\frac{1}{q}=\frac{1}{p}-\frac{\alpha}{n}$ and $\frac{\lambda}{p}=\frac{\mu}{q}$, then $\frac{\alpha}{n}+\frac{\lambda-1}{p}=\frac{\lambda}{p}-\left(\frac{1}{p}-\frac{\alpha}{n}\right)=\frac{\mu}{q}-\frac{1}{q}$ and

$$
\int_{u}^{\infty} t^{\alpha / n} \Phi^{-1}\left(t^{\lambda-1}\right) \frac{d t}{t}=\frac{q}{1-\mu} u^{\frac{\mu-1}{q}}=\frac{q}{1-\mu} \Psi^{-1}\left(u^{\mu-1}\right),
$$


that is, the estimate (15) holds. Also estimate (16) holds since for all $u, r>0$

$$
\begin{aligned}
\int_{u}^{\infty} t^{\frac{\alpha}{n}} \Phi^{-1}\left(\frac{r^{\lambda}}{t}\right) \frac{d t}{t} & =r^{\frac{\lambda}{p}} \int_{u}^{\infty} t^{\frac{\alpha}{n}-\frac{1}{p}} \frac{d t}{t}=\frac{r^{\frac{\lambda}{p}}}{\frac{1}{p}-\frac{\alpha}{n}} u^{\frac{\alpha}{n}-\frac{1}{p}} \\
& =q r^{\frac{\mu}{q}} u^{-1 / q}=q \Psi^{-1}\left(\frac{r^{\mu}}{u}\right) .
\end{aligned}
$$

From the Theorem 3 we get the Spanne-Peetre type result proved in [12, Proposition 1.1], that is, the Riesz potential $I_{\alpha}$ is bounded from $M^{p, \lambda}(0)$ to $M^{q, \mu}(0)$ under the conditions $1<p<\frac{n(1-\lambda)}{\alpha}, 0 \leq \lambda<1, \frac{1}{q}=\frac{1}{p}-\frac{\alpha}{n}$ and $\frac{\lambda}{p}=\frac{\mu}{q}$.

Remark 5 It is easy to see that for $0 \leq \lambda<1$ if $\Phi_{1}, \Phi_{2}$ are two Orlicz functions and there exists a constant $k>0$ such that $\Phi_{2}(u) \leq \Phi_{1}(k u)$ for all $u>0$, then $\|f\|_{\Phi_{2}, \lambda, A} \leq k\|f\|_{\Phi_{1}, \lambda, A}$ provided the right side is finite. Furthemore, $M^{\Phi_{1}, \lambda}\left(\mathbb{R}^{n}\right) \stackrel{k}{\hookrightarrow}$ $M^{\Phi_{2}, \lambda}\left(\mathbb{R}^{n}\right)$ and $M^{\Phi_{1}, \lambda}(0) \stackrel{k}{\hookrightarrow} M^{\Phi_{2}, \lambda}(0)$. Hence it follows that if two Orlicz functions $\Phi_{1}, \Phi_{2}$ are equivalent, i.e. there exist positive constants $k_{1}, k_{2}$ such that $\Phi_{1}\left(k_{1} u\right) \leq$ $\Phi_{2}(u) \leq \Phi_{1}\left(k_{2} u\right)$ for all $u>0$, then $M^{\Phi_{1}, \lambda}\left(\mathbb{R}^{n}\right)=M^{\Phi_{2}, \lambda}\left(\mathbb{R}^{n}\right)$ and $M^{\Phi_{1}, \lambda}(0)=$ $M^{\Phi_{2}, \lambda}(0)$ with equivalent norms.

Example 2 Let $0<\alpha<n, 0 \leq \lambda<1,1<p<\frac{n(1-\lambda)}{\alpha}, a>0$ and

$$
\Phi^{-1}(u)=\left\{\begin{array}{ll}
u^{\frac{1}{p}} & \text { for } 0 \leq u \leq 1, \\
u^{\frac{1}{p}}(1+\ln u)^{-a} & \text { for } u \geq 1,
\end{array} \quad \Psi^{-1}(u)=u^{\frac{1}{q}} \text { with } 1<p<q<\infty .\right.
$$

If $\frac{1}{q}=\frac{1}{p}-\frac{\alpha}{n}, \frac{\lambda}{p}=\frac{\mu}{q}$, then condition (15) is satisfied. Really, for $u \geq 1$ we have equality as in the Example 1 . If $0<u<1$, then using the fact that function $\left(1+\ln t^{\lambda-1}\right)^{-a}$ is strictly increasing of variable $t$ on $(0,1]$, we get $\left(1+\ln t^{\lambda-1}\right)^{-a} \leq 1$ for $0<t \leq 1$ and so

$$
\begin{aligned}
\int_{u}^{\infty} t^{\alpha / n} \Phi^{-1}\left(t^{\lambda-1}\right) \frac{d t}{t} & =\int_{u}^{1} t^{\frac{\alpha}{n}+\frac{\lambda-1}{p}}\left(1+\ln t^{\lambda-1}\right)^{-a} \frac{d t}{t}+\int_{1}^{\infty} t^{\frac{\alpha}{n}+\frac{\lambda-1}{p}} \frac{d t}{t} \\
& \leq \int_{u}^{\infty} t^{\frac{\alpha}{n}+\frac{\lambda-1}{p}} \frac{d t}{t}=\frac{u^{\frac{\alpha}{n}+\frac{\lambda-1}{p}}}{\frac{1-\lambda}{p}-\frac{\alpha}{n}}=\frac{q}{1-\mu} u^{\frac{\mu-1}{q}} \\
& =\frac{q}{1-\mu} \Psi^{-1}\left(u^{\mu-1}\right),
\end{aligned}
$$

that is, the estimate (15) holds. Next, we consider condition (16). If $u \geq r^{\lambda}$, then

$$
\int_{u}^{\infty} t^{\frac{\alpha}{n}} \Phi^{-1}\left(\frac{r^{\lambda}}{t}\right) \frac{d t}{t}=r^{\frac{\lambda}{p}} \int_{u}^{\infty} t^{\frac{\alpha}{n}-\frac{1}{p}} \frac{d t}{t}=\frac{r^{\frac{\lambda}{p}}}{\frac{1}{p}-\frac{\alpha}{n}} u^{\frac{\alpha}{n}-\frac{1}{p}}=q r^{\frac{\mu}{q}} u^{-\frac{1}{q}}=q \Psi^{-1}\left(\frac{r^{\mu}}{u}\right)
$$


Let now $0<u<r^{\lambda}$. Then, $\left(1+\ln \frac{r^{\lambda}}{t}\right)^{-a} \leq 1$ as an increasing function of $t$ on $\left(0, r^{\lambda}\right]$ and since $u<t \leq r^{\lambda}$, we have

$$
\begin{aligned}
\int_{u}^{\infty} t^{\frac{\alpha}{n}} \Phi^{-1}\left(\frac{r^{\lambda}}{t}\right) \frac{d t}{t} & =r^{\frac{\lambda}{p}} \int_{u}^{r^{\lambda}} t^{\frac{\alpha}{n}-\frac{1}{p}}\left(1+\ln \frac{r^{\lambda}}{t}\right)^{-a} \frac{d t}{t}+r^{\frac{\lambda}{p}} \int_{r^{\lambda}}^{\infty} t^{\frac{\alpha}{n}-\frac{1}{p}} \frac{d t}{t} \\
& \leq r^{\frac{\lambda}{p}} \int_{u}^{r^{\lambda}} t^{\frac{\alpha}{n}-\frac{1}{p}} \frac{d t}{t}+r^{\frac{\lambda}{p}} \int_{r^{\lambda}}^{\infty} t^{\frac{\alpha}{n}-\frac{1}{p}} \frac{d t}{t} \\
& =r^{\frac{\lambda}{p}} \int_{u}^{\infty} t^{\frac{\alpha}{n}-\frac{1}{p}} \frac{d t}{t}=\frac{r^{\frac{\lambda}{p}}}{\frac{1}{p}-\frac{\alpha}{n}} u^{\frac{\alpha}{n}-\frac{1}{p}}=q r^{\frac{\mu}{q}} u^{-\frac{1}{q}}=q \Psi^{-1}\left(\frac{r^{\mu}}{u}\right),
\end{aligned}
$$

that is, the estimate (16) holds. The function $\Phi^{-1}$ is increasing, unbounded, obviously concave on $(0,1)$ and concave for large $u$. Therefore, there exists a concave function on $(0, \infty)$ which is equivalent to $\Phi^{-1}$ and so $\Phi$ is equivalent to an Orlicz function. Also we have equivalence

$$
\Phi(u) \approx \begin{cases}u^{p} & \text { for } 0 \leq u \leq 1, \\ u^{p}(1+\ln u)^{a p} & \text { for } u \geq 1\end{cases}
$$

Moreover, since

$$
s_{\Phi^{-1}}(t)= \begin{cases}t^{1 / p}(1-\ln t)^{a} & \text { for } 0<t \leq 1 \\ t^{1 / p} & \text { for } t \geq 1\end{cases}
$$

it follows that the Matuszewska-Orlicz index $\beta_{\Phi^{-1}}=\frac{1}{p}$ and so $1=\frac{1}{\beta_{\Phi^{*}}}+\frac{1}{\alpha_{\Phi}}=$ $\frac{1}{\beta_{\Phi^{*}}}+\beta_{\Phi^{-1}}=\frac{1}{\beta_{\Phi^{*}}}+\frac{1}{p}$ or $\beta_{\Phi^{*}}=\frac{p}{p-1}<\infty$, which means that $\Phi^{*} \in \Delta_{2}$ (for definitions and properties of indices - see [26, pp. 87-89]). Thus, by Remark 5, the space $M^{\Phi, \lambda}(0)$ is a Banach space and by Theorem 3 the Riesz potential $I_{\alpha}$ is bounded from $M^{\Phi, \lambda}(0)$ to $M^{\Psi, \mu}(0)=M^{q, \mu}(0)$.

Example 3 Let $0<\alpha<n, 0 \leq \lambda<1,1<p<\frac{n(1-\lambda)}{\alpha}, 0 \leq b \leq a$ and

$$
\Phi^{-1}(u)=u^{\frac{1}{p}}(1+|\ln u|)^{-a} \text { and } \Psi^{-1}(u)=u^{\frac{1}{q}}(1+|\ln u|)^{b} \text { for } u>0 .
$$

If $\frac{1}{q}=\frac{1}{p}-\frac{\alpha}{n}, \frac{\lambda}{p}=\frac{\mu}{q}$, then conditions (a), (b) of Theorem 2(i) and (15), (16) are satisfied. The calculations are similar to those in Example 2 so we will omit them here. Observe only that

$$
s_{\Phi^{-1}}(t)=t^{1 / p}(1+|\ln t|)^{a}, s_{\Psi^{-1}}(t)=t^{1 / q}(1+|\ln t|)^{b} .
$$

Then, the functions $\Phi^{-1}, \Psi^{-1}$ are increasing, unbounded and concave near 0 and for large $u$, and so the inverses $\Phi, \Psi$ are equivalent to Orlicz functions. Thus, by Remark 5, the spaces $M^{\Phi, \lambda}(0), M^{\Psi, \mu}(0)$ are Banach spaces and by Theorem 3 the Riesz potential $I_{\alpha}$ is bounded from $M^{\Phi, \lambda}(0)$ to $M^{\Psi, \mu}(0)$. 
Acknowledgements The third author is partially supported by Grant-in-Aid for Scientific Research (C), No. 17K05306, No. 20K03663, Japan Society for the Promotion of Science.

Funding Open access funding provided by Luleå University of Technology.

Open Access This article is licensed under a Creative Commons Attribution 4.0 International License, which permits use, sharing, adaptation, distribution and reproduction in any medium or format, as long as you give appropriate credit to the original author(s) and the source, provide a link to the Creative Commons licence, and indicate if changes were made. The images or other third party material in this article are included in the article's Creative Commons licence, unless indicated otherwise in a credit line to the material. If material is not included in the article's Creative Commons licence and your intended use is not permitted by statutory regulation or exceeds the permitted use, you will need to obtain permission directly from the copyright holder. To view a copy of this licence, visit http://creativecommons.org/licenses/by/4.0/.

\section{References}

1. Adams, D.R.: A note on Riesz potentials. Duke Math. J. 42(4), 765-778 (1975)

2. Alvarez, J., Guzmán-Partida, M., Lakey, J.: Spaces of bounded $\lambda$-central mean oscillation, Morrey spaces, and $\lambda$-central Carleson measures. Collect. Math. 51(1), 1-47 (2000)

3. Alzer, H.: Inequalities for the volume of the unit ball in $\mathbb{R}^{n}$. J. Math. Anal. Appl. 252(1), 353-363 (2000)

4. Burenkov, V.I.: Recent progress in studying the boundedness of classical operators of real analysis in general Morrey-type spaces. I. Eurasian Math. J. 3(3), 11-32 (2012)

5. Burenkov, V.I., Gogatishvili, A., Guliyev, V.S., Mustafayev, R.Ch.: Boundedness of the Riesz potential in local Morrey-type spaces. Potential Anal. 35(1), 67-87 (2011)

6. Burenkov, V.I., Guliyev, H.V.: Necessary and sufficient conditions for boundedness of the maximal operator in local Morrey-type spaces. Studia Math. 163(2), 157-176 (2004)

7. Burenkov, V.I., Jain, P., Tararykova, T.V.: On boundedness of the Hardy operator in Morrey-type spaces. Eurasian Math. J. 2(1), 52-80 (2011)

8. Chen, Y., Lau, K.: Some new classes of Hardy spaces. J. Funct. Anal. 84(2), 255-278 (1989)

9. Chiarenza, F., Frasca, M.: Morrey spaces and Hardy-Littlewood maximal function. Rend. Math. Appl. 7(7), 273-279 (1987)

10. Cianchi, A.: Strong and weak type inequalities for some classical operators in Orlicz spaces. J. London Math. Soc. (2) 60(1), 187-202 (1999)

11. Deringoz, F., Guliyev, V.S., Nakai, E., Sawano, Y., Shi, M.: Generalized fractional maximal and integral operators on Orlicz and generalized Orlicz-Morrey spaces of the third kind. Positivity 23(3), 727-757 (2019)

12. Fu, Z.W., Lin, Y., Lu, S.: $\lambda$-central BMO estimates for commutators of singular integral operators with rough kernels. Acta Math. Sin. (Engl. Ser.) 24(3), 373-386 (2008)

13. García-Cuerva, J.: Hardy spaces and Beurling algebras. J. London Math. Soc. (2) 39(3), 499-513 (1989)

14. García-Cuerva, J., Herrero, M.J.L.: A theory of Hardy spaces assosiated to the Herz spaces. Proc. London Math. Soc. (3) 69(3), 605-628 (1994)

15. Garling, D.J.H.: Inequalities. A Journey into Linear Analysis. Cambridge University Press, Cambridge (2007)

16. Grafakos, L.: Modern Fourier Analysis, 2nd edn. Springer, New York (2009)

17. Guliyev, V. S.: Integral Operators on Function Spaces on the Homogeneous Groups and on Domains in $\mathbb{R}^{n}$. Doctoral Dissertation, Mat. Inst. Steklov, Moscow, 329 pp. (in Russian) (1994)

18. Guliyev, V. S.: Function Spaces, Integral Operators and Two Weighted Inequalities on Homogeneous Groups. Some Applications. Casioglu, Baku, 332 pp. (in Russian) (1999)

19. Guliyev, V. S., Deringoz, F.: On the Riesz potential and its commutators on generalized Orlicz-Morrey spaces. J. Funct. Spaces, Article ID 617414, 11 pp (2014)

20. Guliyev, V. S., Deringoz, F., Hasanov, S.: Riesz potential and its commutators on Orlicz spaces. J. Inequal. Appl., Paper No. 75, 18 pp (2017) 
21. Gunawan, H., Hakim, D.I., Idris, M.: Proper inclusions of Morrey spaces. Glas. Mat. Ser. III 53(73), 143-151 (2018)

22. Guzmán-Partida, M.: A note on some operators acting on central Morrey spaces. Mat. Vesnik 70(2), 155-160 (2018)

23. Komori-Furuya, Y., Sato, E.: Fractional integral operators on central Morrey spaces. Math. Inequal. Appl. 20(3), 801-813 (2017)

24. Krasnoselskii, M. A., Rutickii, Ja. B.: Convex Functions and Orlicz Spaces. Noordhoff, Groningen (1961)

25. Kufner, A., John, O., Fucik, S.: Function Spaces, Noordhoff International Publishing. Leyden; Academia, Prague (1977)

26. Maligranda, L.: Orlicz Spaces and Interpolation. Seminars in Math. 5, Universidade Estadual de Campinas, Campinas (1989)

27. Maligranda, L.: Hidegoro Nakano (1909-1974) - on the centenary of his birth, in: "Banach and Function Spaces III" (ISBFS 2009, Kitakyushu, Sept. 14-17, 2009), Yokohama Publ., Yokohama 2011, 99-171

28. Maligranda, L., Matsuoka, K.: Maximal function in Beurling-Orlicz and central Morrey-Orlicz spaces. Colloq. Math. 138(2), 165-181 (2015)

29. Maligranda, L., Matsuoka, K.: Calderón-Zygmund singular integrals in central Morrey-Orlicz spaces. Tohoku Math. J. (2) 72(2), 235-259 (2020)

30. Matsuoka, K., Nakai, E.: Fractional integral operators on $B^{p, \lambda}$ with Morrey-Campanato norms. in: "Function Spaces IX" (Krakow, July 6-11, 2009), Banach Center Publ. 92, 249-264 (2011)

31. Mizuta, Y.: Potential Theory in Euclidean Spaces. Gakkōtosho, Tokyo (1996)

32. Nakai, E.: On generalized fractional integrals. Taiwanese J. Math. 5(3), 587-602 (2001)

33. Nakai, E.: On generalized fractional integrals in the Orlicz spaces on spaces of homogeneous type. Sci. Math. Jpn. 54(3), 473-487 (2001)

34. Nakai, E.: Generalized fractional integrals on Orlicz-Morrey spaces. in: "Banach and Function Spaces" (ISBFS 2003, Kitakyushu, October 2-4, 2003), Yokohama Publ., Yokohama, 323-333 (2004)

35. Nakai, E.: Orlicz-Morrey spaces and the Hardy-Littlewood maximal function. Studia Math. 188(3), 193-221 (2008)

36. Peetre, J.: On the theory of $\mathcal{L}_{p, \lambda}$ spaces. J. Funct. Anal. 4, 71-87 (1969)

37. Rafeiro, H., Samko, S.: Coincidence of variable exponent Herz spaces with variable exponent Morrey type spaces and boundedness of sublinear operators in these spaces. Potential Anal. 56(3), 437-457 (2022)

38. Rao, M.M., Ren, Z.D.: Theory of Orlicz Spaces. Marcel Dekker, New York (1991)

39. Sawano, Y., Sugano, S., Tanaka, H.: Orlicz-Morrey spaces and fractional operators. Potential Anal. 36(4), 517-556 (2012)

40. Stein, E.M.: Singular Integrals and Differentiability Properties of Functions. Princeton University Press, Princeton, NJ (1970)

41. Torchinsky, A.: Real-Variable Methods in Harmonic Analysis. Academic Press, Orlando (1986)

42. Ziemer, W.P.: Weakly Differentiable Functions. Springer-Verlag, New York (1989)

Publisher's Note Springer Nature remains neutral with regard to jurisdictional claims in published maps and institutional affiliations. 